\title{
Constraints on sneutrino dark matter from LHC Run 1
}

\author{
Chiara Arina, ${ }^{a, b}$ Maria Eugenia Cabrera Catalan, ${ }^{b, c, d}$ Sabine Kraml, ${ }^{e}$ \\ Suchita Kulkarni ${ }^{e, f}$ and Ursula Laa ${ }^{e, f}$
}

${ }^{a}$ Institut d'Astrophysique de Paris, 98bis Boulevard Arago, F-75014 Paris, France

${ }^{b}$ GRAPPA Institute, University of Amsterdam, Science Park 904, 1090 GL Amsterdam, Netherlands

${ }^{c}$ Instituto de Física, Universidade de São Paulo, São Paulo, Brazil

${ }^{d}$ Instituto de Física Teórica, IFT-UAM/CSIC, U.A.M. Cantoblanco, 28049 Madrid, Spain

${ }^{e}$ Laboratoire de Physique Subatomique et de Cosmologie, Université Grenoble-Alpes, CNRS/IN2P3, 53 Avenue des Martyrs, F-38026 Grenoble, France

${ }^{f}$ Institut für Hochenergiephysik, Österreichische Akademie der Wissenschaften, Nikolsdorfer Gasse 18, 1050 Wien, Austria

E-mail: carina@uva.nl, mcabrera@if.usp.br, sabine.kraml@lpsc.in2p3.fr, suchita.kulkarni@oeaw.ac.at, ursula.laa@lpsc.in2p3.fr

ABSTRACT: A mostly right-handed sneutrino as the lightest supersymmetric particle (LSP) is an interesting dark matter candidate, leading to LHC signatures which can be quite distinct from those of the conventional neutralino LSP. Using SMODELS v1.0.1 for testing the model against the limits published by ATLAS and CMS in the context of so-called Simplified Model Spectra (SMS), we investigate to what extent the supersymmetry searches at Run 1 of the LHC constrain the sneutrino-LSP scenario. Moreover, we discuss the most relevant topologies for which no SMS results are provided by the experimental collaborations but which would allow to put more stringent constraints on sneutrino LSPs. These include, for instance, the mono-lepton signature which should be particularly interesting to consider at Run 2 of the LHC.

Keywords: Supersymmetry Phenomenology

ARXIV EPRINT: 1503.02960 


\section{Contents}

1 Introduction $\quad 1$

2 The MSSM+RN model $\quad 3$

3 Numerical procedure $\quad 4$

3.1 Sampling method over the model parameters 4

$\begin{array}{lll}3.2 & \text { Deriving LHC constraints with SModelS }\end{array}$

$\begin{array}{llr}4 & \text { Results } & 8\end{array}$

5 Conclusions 16

A Validity of slepton search results for chargino-pair production with decay $\begin{array}{lr}\text { into lepton+sneutrino } & 18\end{array}$

B Lifetimes of long-lived particles $\quad 20$

\section{Introduction}

Before the start of data taking at the LHC, the common perception was that supersymmetry (SUSY), if it has anything to do with stabilizing the electroweak (EW) scale, would be discovered quickly, while Higgs physics would need to wait for rather high statistics. In reality, quite the opposite has happened: a Higgs boson has been found [1, 2], but there is still no sign of SUSY - or of any new physics beyond the Standard Model (BSM) whatsoever.

Indeed, the searches at Run 1 of the LHC at centre-of-mass energies of $7-8 \mathrm{TeV}$ have pushed the mass limits of SUSY particles quite high already, well above $1 \mathrm{TeV}$ for 1 st/2nd generation squarks and gluinos [3, 4]. Scenarios with high-scale [5], split [6-8] or at least spread [9] SUSY are thus becoming increasingly popular in the literature. It should be kept in mind, however, that the current LHC limits sensitively depend on the presence of particular decay modes, and are considerably weakened in case of compressed [10] or stealth [11] spectra. Besides, the squark/gluino mass limits vanish completely in case the neutralino LSP is heavier than about $600 \mathrm{GeV}$.

It should also be kept in mind that the SUSY mass limits depend sensitively on the nature of the LSP. Most experimental analyses indeed assume that the LSP is the lightest neutralino of the Minimal Supersymmetric Standard Model (MSSM). A particularly interesting alternative, and the subject of this paper, is a mainly right-handed ( $\mathrm{RH})$ mixed sneutrino in the MSSM augmented by a RH neutrino superfield $[12,13]$. This case is well motivated by two basic problems: the origin of neutrino masses and the nature of dark 
matter (DM). Its LHC signatures can be quite distinct from those of the conventional neutralino LSP.

The left-handed (LH) sneutrino of the MSSM is excluded as the LSP and as a DM candidate because it has a non-zero hypercharge: its couplings to the $Z$ boson makes it annihilate too efficiently in the early Universe, and hence its final relic abundance is lower than the value $\Omega_{\mathrm{DM}} h^{2}$ measured by the WMAP and Planck satellites [14, 15]. Very stringent limits come moreover from direct DM detection experiments: the $\tilde{\nu}_{L}$ scattering off nuclei is mediated by $t$-channel $Z$ boson exchange, giving a spin-independent (SI) cross section of order $10^{-39} \mathrm{~cm}^{2}$ - a value excluded already a decade ago for DM particles heavier than $10 \mathrm{GeV}$. A light $\tilde{\nu}_{L}$ with mass below $m_{Z} / 2$ is also excluded by the $Z$ invisible width.

The picture changes dramatically if we include in the MSSM a RH neutrino superfield (MSSM+RN from here on), which gives rise to Dirac neutrino masses. Besides the RH neutrino, the superfield also contains a scalar field, the RH sneutrino $\tilde{N}$ (strictly speaking this is a right-chiral field, but we use the $\mathrm{RH}$ notation for simplicity). This field, if at $\mathrm{TeV}$ scale, can mix with the LH partner $\tilde{\nu}_{L}$ and yield a mostly RH sneutrino LSP as a viable thermal DM candidate $[12,13] .^{1}$

The phenomenology of this model was investigated in detail in [13, 21, 22]. Indirect detection and cosmology were discussed in [20, 23, 24], and LHC signatures in [25-27] (see also [28-30] for related LHC studies). Reference [27] in fact gave an update of the status of the sneutrino as DM after the Higgs mass measurements, by exploring the SUSY parameter space with the soft breaking terms fixed at the grand unification (GUT) scale, and assessing also the impact of the most recent exclusion bound for DM direct searches from LUX [31].

In this paper, we extend the work of [27] by investigating to what extent the results from SUSY searches at Run 1 of the LHC, published in terms of so-called Simplified Model Spectra (SMS) limits ${ }^{2}$ constrain the sneutrino-LSP scenario. Moreover, we discuss the most promising topologies for which no SMS results exist but would enhance the LHC sensitivity to sneutrino DM. To this aim, we make use of the SModeLS v1.0.1 package [34-36] to compare the predictions of the MSSM+RN model against the SMS limits published by ATLAS and CMS. The strengths of SMODELS v1.0.1 are that it 1.) automatically decomposes the signal of an arbitrary SUSY spectrum into all its SMS-equivalent topologies, and 2.) includes a large database of more than 60 SMS results from ATLAS and CMS SUSY searches. This allows us to test the limits from a large variety of searches and at the same time draw conclusions about which additional topologies should be considered.

The paper is organised as follows. After briefly defining the MSSM+RN in section 2 we describe the numerical procedure in section 3. In particular in 3.1 we explain the sampling method and the constraints implemented in the model likelihood function, while in 3.2 we describe the application of SMODELS v1.0.1 to the MSSM+RN. Our numerical results are presented in section 4 , and the conclusions in section 5. Two appendices contain

\footnotetext{
${ }^{1}$ Pure right-handed sterile sneutrinos can also be viable (non-thermal, depending on the model) DM candidates, as discussed e.g. in [16-20].

${ }^{2}$ Simplified Models are effective-Lagrangian descriptions involving only a small number of new particles. They were designed as a useful tool for the characterization of new physics, see e.g. [32, 33].
} 
some interesting supplementary material. Appendix A discusses the validity of applying SMS results from slepton searches (dilepton signature) to chargino-pair production followed by decays into leptons and sneutrinos. Appendix B gives some details on scenarios with long-lived heavy charged particles, in particular gluinos or stops, which so far cannot be constrained by SMS results.

\section{The MSSM+RN model}

We use the MSSM+RN model as defined in [12, 13, 24]. (The model used in [21, 22, 26] differs only slightly in notation.) The superpotential for Dirac RH neutrino superfield is given by

$$
W=\epsilon_{i j}\left(\mu \hat{H}_{i}^{u} \hat{H}_{j}^{d}-Y_{l}^{I J} \hat{H}_{i}^{d} \hat{L}_{j}^{I} \hat{R}^{J}+Y_{\nu}^{I J} \hat{H}_{i}^{u} \hat{L}_{j}^{I} \hat{N}^{J}\right),
$$

where $Y_{\nu}^{I J}$ is a matrix in flavor space (which we choose to be real and diagonal), from which the mass of neutrinos are obtained as $m_{D}^{I}=v_{u} Y_{\nu}^{I I}$. Note that lepton-number violating terms are absent in this scheme. The additional scalar fields contribute with new terms in the soft-breaking potential

$$
V_{\text {soft }}=\left(M_{L}^{2}\right)^{I J} \tilde{L}_{i}^{I *} \tilde{L}_{i}^{J}+\left(M_{N}^{2}\right)^{I J} \tilde{N}^{I *} \tilde{N}^{J}-\left[\epsilon_{i j}\left(\Lambda_{l}^{I J} H_{i}^{d} \tilde{L}_{j}^{I} \tilde{R}^{J}+\Lambda_{\nu}^{I J} H_{i}^{u} \tilde{L}_{j}^{I} \tilde{N}^{J}\right)+\text { h.c. }\right]
$$

where both matrices $M_{N}^{2}$ and $\Lambda_{\nu}^{I J}$ are real and diagonal, $M_{N}^{2}=\operatorname{diag}\left(m_{N^{k}}^{2}\right)$ and $\Lambda_{\nu}^{I J}=$ $\operatorname{diag}\left(A_{\tilde{\nu}}^{k}\right)$, with $k=e, \mu, \tau$ being the flavor index. In the sneutrino interaction basis, defined by the vector $\Phi^{\dagger}=\left(\tilde{\nu}_{L}^{*}, \tilde{N}^{*}\right)$, the sneutrino mass potential is

$$
V_{\text {mass }}^{k}=\frac{1}{2} \Phi_{L R}^{\dagger} \mathcal{M}_{L R}^{2} \Phi_{L R}
$$

with the squared-mass matrix $\mathcal{M}_{L R}^{2}$

$$
\mathcal{M}_{L R}^{2}=\left(\begin{array}{cc}
m_{L^{k}}^{2}+\frac{1}{2} m_{Z}^{2} \cos (2 \beta)+m_{D}^{2} & \frac{1}{\sqrt{2}} A_{\tilde{\nu}}^{k} v \sin \beta-\mu m_{D} / \tan \beta \\
\frac{1}{\sqrt{2}} A_{\tilde{\nu}}^{k} v \sin \beta-\mu m_{D} / \tan \beta & m_{N^{k}}^{2}+m_{D}^{2}
\end{array}\right) .
$$

Here, $m_{L^{k}}^{2}$ are the soft mass terms for the three $\mathrm{SU}(2)$ leptonic doublets, $\tan \beta=v_{u} / v_{d}$ and $v^{2}=v_{u}^{2}+v_{d}^{2}=(246 \mathrm{GeV})^{2}$, with $v_{u, d}$ the usual Higgs vacuum expectation values (vevs). The Dirac neutrino mass $m_{D}$ is small and can be safely neglected.

The off-diagonal term determines the mixing of the LH and RH fields. If $A_{\tilde{\nu}}^{k}=\eta Y_{\nu}$, that is if the trilinear term is aligned to the neutrino Yukawa, this term is certainly very small as compared to the diagonal entries and is therefore negligible. However, $A_{\tilde{\nu}}^{k}$ can in general be a free parameter and may naturally be of the order of the other entries of the matrix $[12,13]$, thus inducing a sizable mixing among the interaction eigenstates. The sneutrino mass eigenstates are then given by

$$
\left(\begin{array}{l}
\tilde{\nu}_{k_{1}} \\
\tilde{\nu}_{k_{2}}
\end{array}\right)=\left(\begin{array}{cc}
-\sin \theta_{\tilde{\nu}}^{k} & \cos \theta_{\tilde{\nu}}^{k} \\
\cos \theta_{\tilde{\nu}}^{k} & \sin \theta_{\tilde{\nu}}^{k}
\end{array}\right)\left(\begin{array}{c}
\tilde{\nu}_{L}^{k} \\
\tilde{N}^{k}
\end{array}\right)
$$


The relevant parameters at the EW scale for the sneutrino sector are the two mass eigenvalues $m_{\tilde{\nu}_{k_{1}}}$ and $m_{\tilde{\nu}_{k_{2}}}$ and the mixing angle $\theta_{\tilde{\nu}}^{k}$, related to the $A_{\tilde{\nu}}^{k}$ term via

$$
\sin 2 \theta_{\tilde{\nu}}^{k}=\sqrt{2} \frac{A_{\tilde{\nu}}^{k} v \sin \beta}{\left(m_{\tilde{\nu}_{k 2}}^{2}-m_{\tilde{\nu}_{k 1}}^{2}\right)} .
$$

The sneutrino coupling to the $Z$ boson, which does not couple to $\mathrm{SU}(2)_{L}$ singlets, is largely reduced by a sizeable mixing. This has a relevant impact on the sneutrino phenomenology, as discussed in, e.g., refs. [13, 21, 24, 37, 38].

The renormalization group equations (RGEs) are modified by the new singlet superfields $\hat{N}$ as

$$
\begin{aligned}
\frac{\mathrm{d} m_{N^{k}}^{2}}{\mathrm{~d} \ln \mu} & =\frac{4}{16 \pi^{2}}\left(A_{\tilde{\nu}}^{k}\right)^{2}, \\
\frac{\mathrm{d} m_{L^{k}}^{2}}{\mathrm{~d} \ln \mu} & =(\text { MSSM terms })+\frac{2}{16 \pi^{2}}\left(A_{\tilde{\nu}}^{k}\right)^{2}, \\
\frac{\mathrm{d} A_{\tilde{\nu}}^{k}}{\mathrm{~d} \ln \mu} & =\frac{2}{16 \pi^{2}}\left(-\frac{3}{2} g_{2}^{2}-\frac{3}{10} g_{1}^{2}+\frac{3}{2} Y_{t}^{2}+\frac{1}{2} Y_{\tau}^{2}\right) A_{\tilde{\nu}}^{k}, \\
\frac{\mathrm{d} m_{H_{u}}^{2}}{\mathrm{~d} \ln \mu} & =(\text { MSSM terms })+\sum_{k=e, \mu, \tau} \frac{2}{16 \pi^{2}}\left(A_{\tilde{\nu}}^{k}\right)^{2},
\end{aligned}
$$

with $\mu$ being the renormalization scale, $g_{2}$ and $g_{1}$ the $\mathrm{SU}(2)$ and $\mathrm{U}(1)$ gauge couplings, $Y_{t, \tau}$ the top and $\tau$ Yukawa respectively. Notice that the RH soft mass receives corrections only from the trilinear term, which affects as well the running of the LH part, as recognized in $[21,26]$.

By neglecting all lepton Yukawas but $Y_{\tau}$ in the RGEs and by assuming common scalar masses and trilinear couplings for all flavors, the sneutrino tau, $\tilde{\nu}_{\tau_{1}}$, ends up to be the lightest one among the three sneutrino flavors and hence the LSP, while $\tilde{\nu}_{e_{1}}=\tilde{\nu}_{\mu_{1}}$. Note that it frequently happens that the mass splitting between $\tilde{\nu}_{\tau_{1}}$ and $\tilde{\nu}_{e_{1}, \mu_{1}}$ is smaller than $5 \mathrm{GeV}$, which means that regarding collider phenomenology they are practically degenerate. This will be discussed in more detail in section 3.2.

\section{Numerical procedure}

\subsection{Sampling method over the model parameters}

For definiteness, we study the MSSM+RN with soft terms defined at a high scale $M \sim$ $M_{\mathrm{GUT}}$ as in [27]. Allowing for non-universalities in the gaugino and scalar sectors, our set of free parameters is

$$
M_{1}, M_{2}, M_{3}, m_{L}, m_{R}, m_{N}, m_{Q}, m_{H}, A_{l}, A_{\tilde{\nu}}, A_{q}, \tan \beta, \operatorname{sgn} \mu .
$$

Here the $M_{i}$ are the gaugino masses, $m_{L}, m_{R}, m_{N}$ are the charged slepton and sneutrino masses (equal for all flavors), $m_{Q}$ is a common squark mass parameter, $m_{H} \equiv m_{H_{u}}=m_{H_{d}}$ denotes the common entry for the two Higgs doublet masses, and $A_{l}$ and $A_{q}$ are the scalar 


\begin{tabular}{|c|c|c|c|}
\hline & Observable & Value/Constraint & Ref. \\
\hline Measurements & $m_{h}$ & $125.85 \pm 0.4 \mathrm{GeV}(\exp ) \pm 4 \mathrm{GeV}$ (theo) & {$[1,2]$} \\
& $\mathrm{BR}\left(B \rightarrow X_{s} \gamma\right) \times 10^{4}$ & $3.55 \pm 0.24 \pm 0.09(\mathrm{exp})$ & {$[39]$} \\
& $\mathrm{BR}\left(B_{s} \rightarrow \mu^{+} \mu^{-}\right) \times 10^{9}$ & $3.2_{-1.2}^{+1.4}(\text { stat })_{-0.3}^{+0.5}(\mathrm{sys})$ & {$[40]$} \\
& $\Omega_{\mathrm{DM}} h^{2}$ & $0.1186 \pm 0.0031(\mathrm{exp}) \pm 20 \%$ (theo $)$ & {$[15]$} \\
\hline Limits & $\Delta \Gamma_{Z}^{\text {invisible }}$ & $<2 \mathrm{MeV}(95 \% \mathrm{CL})$ & {$[41]$} \\
& $\mathrm{BR}(h \rightarrow$ invisible $)$ & $<20 \%(95 \% \mathrm{CL})$ & {$[42]$} \\
& $m_{\tilde{\tau}_{1}^{-}}$ & $>85 \mathrm{GeV}(95 \% \mathrm{CL})$ & {$[43]$} \\
& $m_{\tilde{\chi}_{1}^{+}}, m_{\tilde{e}, \tilde{\mu}}$ & $>101 \mathrm{GeV}(95 \% \mathrm{CL})$ & {$[41]$} \\
& $m_{\tilde{g}}$ & $>308 \mathrm{GeV}(95 \% \mathrm{CL})$ & {$[44]$} \\
& $\sigma_{n}^{\mathrm{SI}}$ & $<\sigma_{\mathrm{LUX}}^{\mathrm{SI}}(90 \% \mathrm{CL})$ & {$[31]$} \\
\hline
\end{tabular}

Table 1. Summary of the observables and constraints used in this analysis.

trilinear couplings for the sleptons and squarks respectively, same for all flavors. The absolute value of $\mu$ is obtained from the minimization of the Higgs potential, leaving only the sign of $\mu$ as a free parameter. The computation of the mass spectrum follows that explained in [27], where all details are provided.

The list of constraints implemented in the model likelihood function is given in table 1. In particular, besides consistency with $B$-physics constraints, we require the Higgs mass $m_{h}$ to be compatible with the ATLAS and CMS measurements $[1,2]$, which we combine by a statistical mean, as obtained in [45]. Its uncertainty is dominated by the theoretical error, estimated to be around $4 \mathrm{GeV}[46]$. We also require that chargino and charged slepton masses fulfill the LEP bounds at $95 \%$ confidence level (CL) - notice that the tau slepton has a slightly less stringent lower bound of $85 \mathrm{GeV}$ [43] as compared to selectrons and smuons - and we include the gluino mass bound from the D0 collaboration [44]. If $\tilde{\nu}_{\tau_{1}}$ is light enough to be produced in $Z$ decay, we require its contribution to the $Z$ invisible decay width to be smaller than $2 \mathrm{MeV}$ [47]. Similarly, when the sneutrino mass is lighter than $m_{h} / 2$, the Higgs can decay invisibly into sneutrino pairs. We require that such decays do not contribute more than $20 \%$ to the Higgs invisible branching ratio [42].

Regarding DM constraints, we require consistency with the measured relic abundance and with the bounds from direct detection experiments (constraints from indirect DM detection are also fulfilled). The experimental error on $\Omega_{\mathrm{DM}} h^{2}$ has become incredibly small due to the Planck measurement [15], while the theoretical one is still large. We use a conservative estimate of the order $20 \%$ [48] for the latter. Furthermore, we enforce the sneutrino SI scattering cross section off nuclei, $\sigma_{n}^{\text {SI }}$, to be compatible with the recent 90\% CL bound from LUX [31].

To evaluate the experimental observables we first computed the supersymmetric particle spectrum with a modified version of SoftSusy [49]. For the computation of the sneutrino relic density and elastic scattering cross section the model has been implemented in FeynRules $[50,51]$, by adding the appropriate term in the superpotential and in the 
soft SUSY breaking potential. We generate output files compatible with CALCHEP in order to use the public code MICROMEGAS_3.2 [52]. The $B$-physics observables are computed by interfacing the program with SUPERIso [53].

The likelihood is constructed in a simple way. For measured quantities, we assume a Gaussian likelihood function with a variance given by combining in quadrature the theoretical and experimental variances. For observables for which only lower or upper limits are available, we use a likelihood modelled as a step function on the $x \%$ CL of the exclusion limit. The total likelihood function is then the product of the individual likelihoods associated to each experimental result. In order to save time in the sampling procedure, the slepton, chargino and gluino mass limits are, however, absorbed into the prior probability density functions: each parameter point generating a mass spectrum that violates one of these bounds is immediately discarded.

Given the likelihood function, we sample the posterior probability density function with the MultiNest algorithm [54-56]. In order to cover all phenomenological interesting classes, we run separate chains that look either for light(ish) EW-inos $\left(m_{\tilde{\chi}_{1}^{ \pm}}<900 \mathrm{GeV}\right)$, light sleptons $\left(m_{\tilde{l}}<600 \mathrm{GeV}\right)$, or for light squark or gluinos $\left(m_{\tilde{q}}<1.5 \mathrm{TeV}\right.$ or $m_{\tilde{g}}<$ $1.5 \mathrm{TeV})$. As for the choice of priors, we always take logarithmic priors on $M_{3}, m_{Q}, A_{Q}, m_{H}$, while we use both logarithmic and flat priors for $M_{1}, M_{2}, m_{L}, m_{R}, m_{N}, A_{L}, A_{\tilde{\nu}}$, $\tan \beta$, the sign of $\mu$ is fixed to +1 (details on the prior ranges are provided in [27]). In particular we perform two chains, one with log and one with flat priors, for each relevant data set: two chains for light EW-inos (these two data sets coincide with the ones used in [27]), two chains for light sleptons and two chains for light squarks or gluinos. In each case, the other masses are left to vary freely from high to low values. The motivation for this is, as mentioned, to cover all potentially interesting cases; the results we will present in section 4 are for all chains combined together.

The sampled points correspond to a 95\% CL in volume of the posterior. (Since in this study we are not interested in statistical statements on the parameter space, we will however not exploit this feature.) The limits imposed by a step function are of course strictly obeyed by all scan points. Moreover, we have checked that none of the individual constraints implemented by a Gaussian gets a large pull in the final sample. In particular, $\mathrm{BR}\left(B \rightarrow X_{s} \gamma\right)$ and $\mathrm{BR}\left(B_{s} \rightarrow \mu^{+} \mu^{-}\right)$are in full agreement with the $95 \%$ CL experimental results $[39,40]$ for all points in the samples.

Once the sampling of the parameter space according to the constraints in table 1 is completed, all the points in the chains are confronted against the LHC Run 1 results by means of SMODELS v1.0.1 as explained in the next subsection.

\subsection{Deriving LHC constraints with SModelS}

SMODELS v1.0.1 [34-36] is designed to decompose the signal of any arbitrary BSM spectrum with a $\mathbb{Z}_{2}$ symmetry into simplified model topologies and test it against the existing LHC bounds in the SMS context. SModelS v1.0.1 uses Pythia 6.4 [57], NLL-fast [58-64] and PySLHA [65], and includes a database of more than 60 SMS results from ATLAS and CMS. The decomposition procedure works "out of the box" for the MSSM+RN model with 

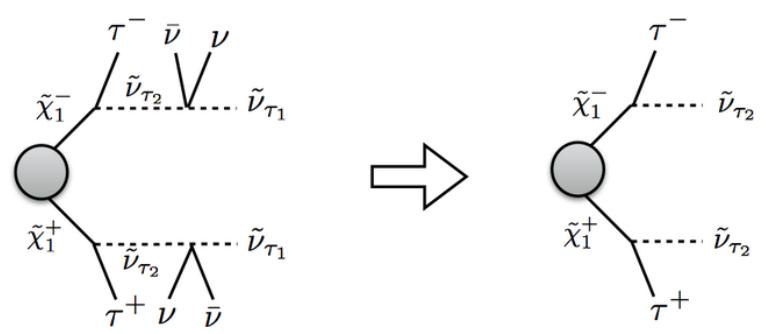

Figure 1. Illustration of "invisible compression" in SMOdELS v1.0.1. The decays of the heavier sneutrino to the lighter one plus neutrinos are discarded in the final topology, leaving the $\tilde{\nu}_{\tau_{2}}$ as an effective LSP.

a sneutrino LSP. Nonetheless some subtleties must be taken care of when processing the MSSM+RN scan points with SMOdELS v1.0.1.

First, the input to SModeLS v1.0.1 can be simulated events or an SLHA [66] file containing the full mass spectrum and decay tables as well as the SUSY production cross sections, $\sigma$, in the format specified at [67]. We choose the latter option. We use the MSSM+RN model implemented in MICROMEGAs_3.2 [52] (see [27]) to compute the decay branching ratios, $\mathcal{B}$. The production cross sections for sleptons and sneutrinos (i.e. the sector modified with respect to the MSSM) are also computed with MICROMEGAs_3.2. For all other production processes, we use the default SMODELS v1.0.1 cross section calculator based on Pythia 6.4 [57] and NLL-fast [58-64]. Electroweak cross sections are thus computed at leading order while strong productions are computed at NLO+NLL order.

Given the information on $\sigma$ and $\mathcal{B}$ in the SLHA files, SModelS v1.0.1 computes $\sigma \times \mathcal{B}$ for each topology that occurs. Here, a topology is characterised by the SM particles originating from each vertex, and the mass vector of the SUSY particles in the decays. In order to avoid dealing with a large number of irrelevant processes, which is expensive in terms of computing time, topologies for which $\sigma \times \mathcal{B}<\sigma_{\text {cut }}$, with $\sigma_{\text {cut }}=0.05 \mathrm{fb}$, are discarded.

When dealing with an arbitrary spectrum of SUSY particles, it is possible that a part of the decay chain leads to completely invisible decays, e.g. a decay of a heavy sneutrino to a lighter one plus neutrinos in the current scenario. In such cases, SModeLS v1.0.1 compresses the invisible part of the decay chain as illustrated in figure 1. All decays to neutrinos appearing after the last visible decay are disregarded, yielding an "effective LSP" for the particular event, which can be different from the true LSP. This procedure is called "invisible compression". Likewise, a neutralino may decay invisibly to a sneutrino and a neutrino; in this case the compressed topology resembles an MSSM topology.

In addition, if the mass gap between mother and daughter particles is small, the decay products will be too soft to be detected at the LHC. This is taken care of by the so-called "mass compression" in SModeLS v1.0.1, discarding any SM particle that come from a vertex for which the mass splitting of the R-odd particles is less than a certain threshold. We use $5 \mathrm{GeV}$ as the minimum required mass difference for the decay products to be visible.

Another comment is in order. The experimental constraints currently implemented in the SMOdELS v1.0.1 database require final states containing missing transverse energy (MET). This means that scenarios with long-lived particles $(c \tau>10 \mathrm{~mm})$ leading to 
signatures with displaced vertices or heavy charged particle tracks cannot be tested with SMODELS v1.0.1. In the MSSM, this problem occurs, e.g., in wino-LSP scenarios where the $\widetilde{\chi}_{1}^{ \pm}$is highly mass-degenerate with the $\widetilde{\chi}_{1}^{0}$ and thus becomes long-lived. In the sneutrino LSP case, not only charginos can be long lived if the mass splitting with the sneutrino is small enough; other possibilities are, e.g., long-lived gluinos or stops, if they are the next-to-lightest SUSY particle (NLSP). We perform a detailed check of all input points to avoid the erroneous application of SMS limits to such cases. Points that have visible decays from long-lived particles or heavy charged particle tracks with cross sections larger than $\sigma_{\text {cut }}$ are discarded. (A brief discussion of such scenarios can be found in appendix B.)

Once the decomposition into SMS topologies, including mass and invisible compression, is completed and the checks that the SMS results actually apply are passed, a given point is confronted against the SMS results in the SMODELS v1.0.1 database. For each experimental constraint that exists, SMODELS v1.0.1 reports among other things the analysis name, the Tx name identifying the topology ${ }^{3}$ the predicted signal cross section for the point under consideration and the 95\% CL experimental upper limit on it. Finally, the ratio $r$ of the signal cross section and the upper limit, $r=\sigma$ (predicted) $/ \sigma$ (excluded), is given, where $\sigma$ effectively means $\sigma \times \mathcal{B}$ or the weight of the topology. A value of $r \geq 1$ means that the input model is likely excluded by the corresponding analysis.

\section{Results}

We now turn to analysing the impact of the LHC searches on the MSSM+RN parameter space. As explained in the previous section, we here consider only points for which the SMS results apply, i.e. we discard points with non-prompt visible decays as well as points with long-lived charged particles. Scanning over the parameter space, we can then distinguish several cases:

- the SMS results in principle apply but no SMS constraints actually exist for the specific topologies of the point — these points will be labelled as not tested ${ }^{4}$

- there exist (one or more) SMS results that test the specific topologies of the point but for each topology the total $\sigma \times \mathcal{B}$ is below the corresponding 95\% CL upper limit - these points will be considered as allowed; and

- at least one topology has a $\sigma \times \mathcal{B}$ equal or above its 95\% CL upper limit $(r \geq 1)-$ these points will be considered as excluded.

Let us start with the question which analyses are the most important ones for constraining the model. To this end, figures 2 and 3 show a breakdown of most constraining analyses in the $\tilde{\nu}_{\tau_{1}}$ versus $\tilde{g}$ and $\tilde{\nu}_{\tau_{1}}$ versus $\tilde{\chi}_{1}^{ \pm}$mass planes, respectively. Looking first at figure 2, we see that (the SMS interpretations of) the hadronic SUSY searches [68-74]

\footnotetext{
${ }^{3}$ The Tx names are explained in the SMS dictionary on http://smodels.hephy.at/wiki/SmsDictionary.

${ }^{4}$ This occurs if no simplified model result exists for the signal topologies of the point considered, but also if the mass vector of a topology lies outside that of the experimental constraint. Moreover, we include here also the points for which all signal topologies are discarded because of $\sigma \times \mathcal{B}<\sigma_{\text {cut }}$.
} 


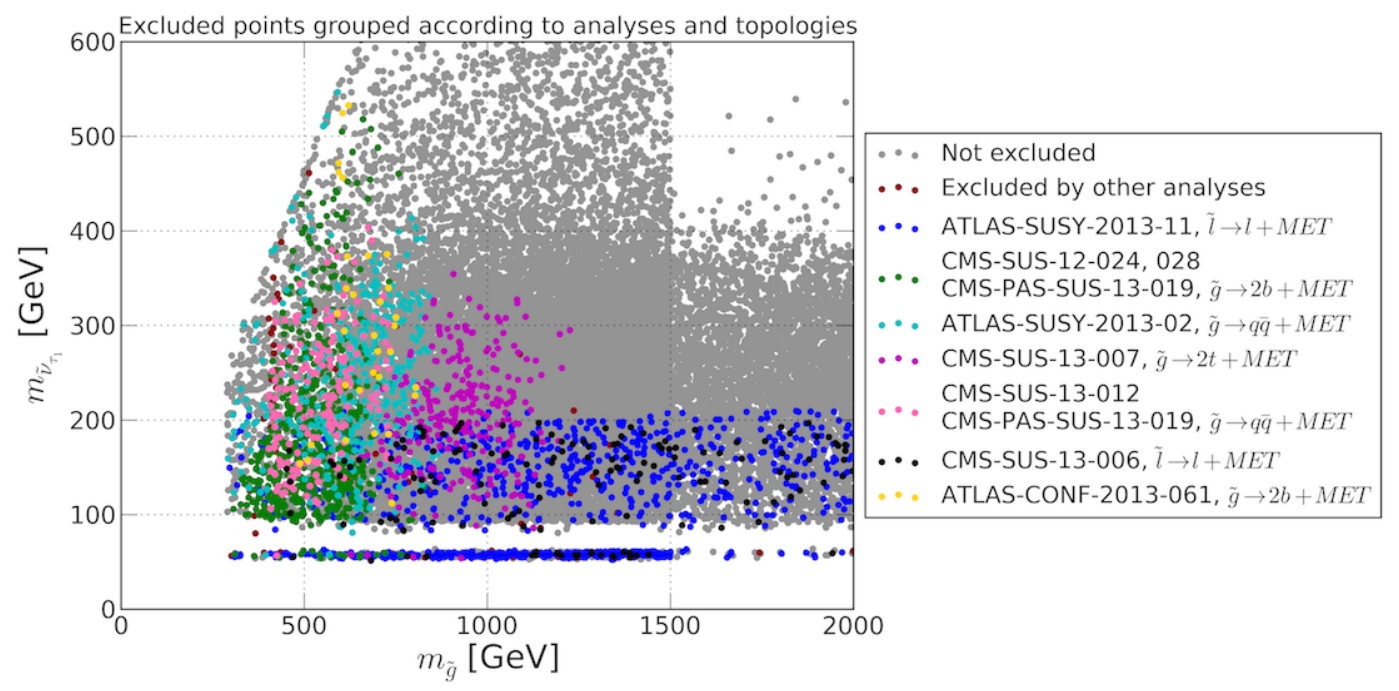

Figure 2. For scan points that are excluded by the SMS limits, we show (in color) the breakdown of most constraining analyses in the $\tilde{\nu}_{\tau_{1}}$ vs. $\tilde{g}$ mass plane. To illustrate the coverage of the parameter space, we also show (in grey) the not excluded or not tested points.

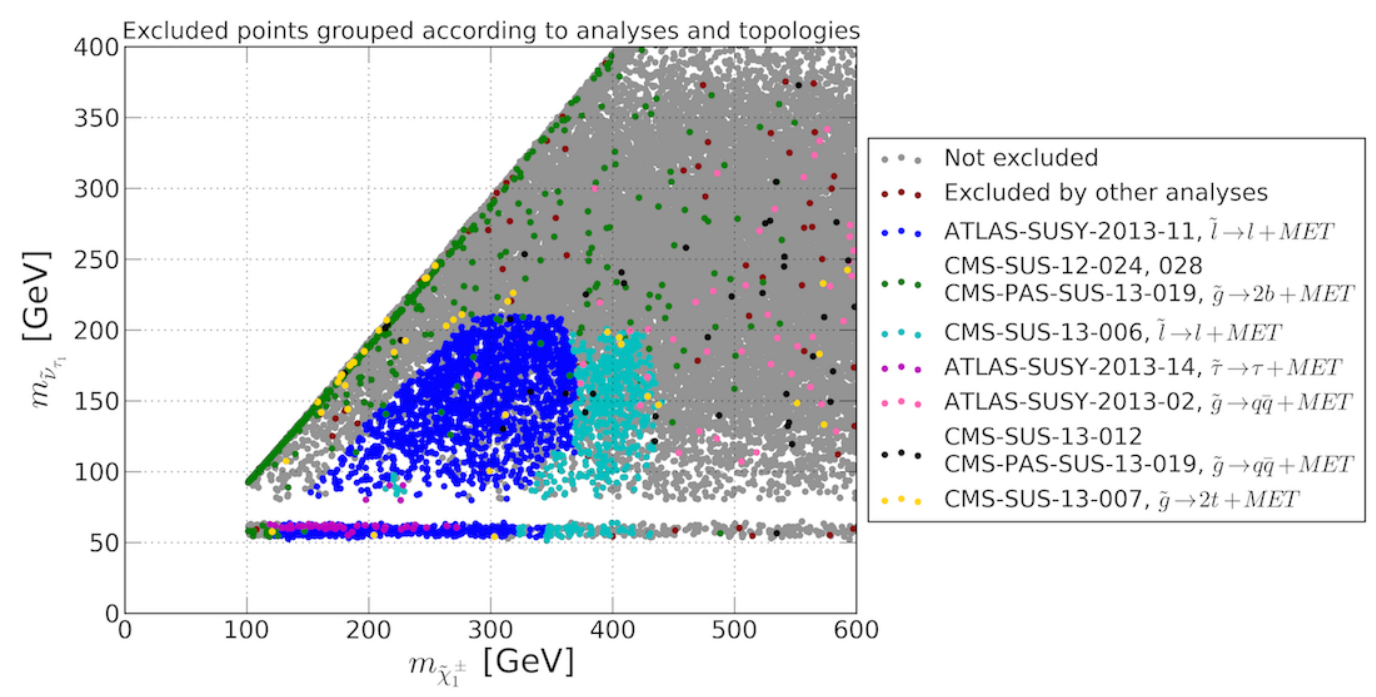

Figure 3. As figure 2 but in the $\tilde{\nu}_{\tau_{1}}$ vs. $\tilde{\chi}_{1}^{ \pm}$mass plane.

are constraining gluino masses up to about $m_{\tilde{g}} \approx 1200 \mathrm{GeV}$ and LSP masses up to about $m_{\tilde{\nu}_{\tau_{1}}} \approx 500 \mathrm{GeV}$. These searches mostly exclude points where either $\tilde{g} \rightarrow b \bar{b} \widetilde{\chi}_{i}^{0}, \tilde{g} \rightarrow t \bar{t} \widetilde{\chi}_{i}^{0}$ or $\tilde{g} \rightarrow q \bar{q} \widetilde{\chi}_{i}^{0}$ decays are dominant, followed by an invisible decay of the neutralino, $\widetilde{\chi}_{i}^{0} \rightarrow \nu \tilde{\nu}$. Moreover, dilepton + MET searches $[75,76]$ exclude sneutrino LSP masses up to about $m_{\tilde{\nu}_{\tau_{1}}} \approx 210 \mathrm{GeV}$, independent of the gluino mass. The process that is constrained here is Drell-Yang production of $\tilde{\chi}_{1}^{+} \tilde{\chi}_{1}^{-}$followed by $\tilde{\chi}_{1}^{ \pm} \rightarrow l^{ \pm} \tilde{\nu}_{l 1}(l=e$ or $\mu)$, with the $\tilde{\nu}_{l 1} \rightarrow \tilde{\nu}_{\tau_{1}}+X$ decay being invisible (because of $X$ being genuinely invisible or very soft). Consequently, in figure 3 we see that chargino masses can be excluded up to about $m_{\widetilde{\chi}_{1}^{ \pm}} \approx 440 \mathrm{GeV}$ by 

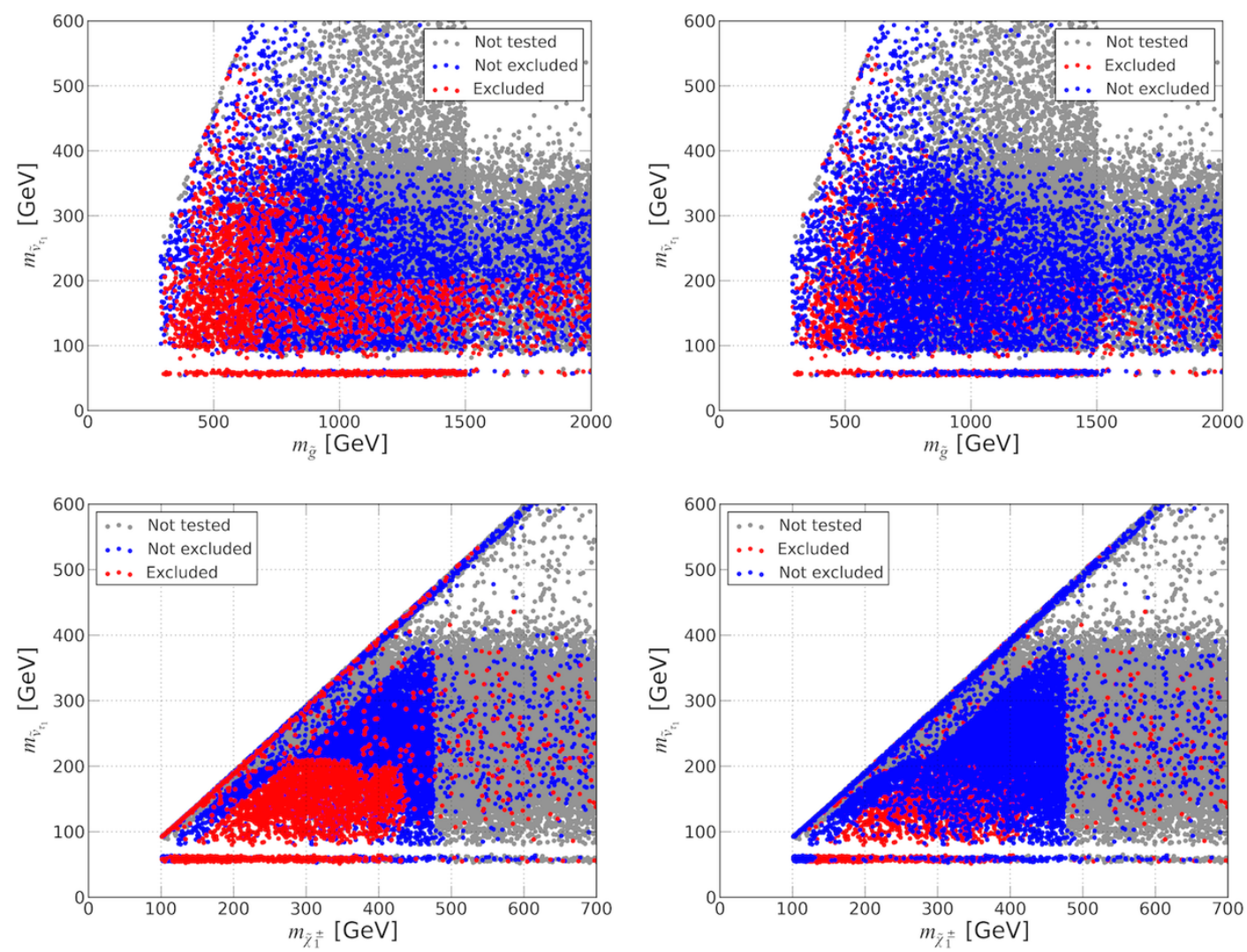

Figure 4. Scatter plots of points for which SMS results apply. The top row shows the $\tilde{\nu}_{\tau_{1}}$ vs. $\tilde{g}$, the bottom row the $\tilde{\nu}_{\tau_{1}}$ vs. $\tilde{\chi}_{1}^{ \pm}$mass plane. In the panels on the left, the points excluded by the SMS constraints (red) are plotted on top of those which are not excluded (blue); in panels on the right this plotting order is inverted. Also shown (in grey) are the "not tested" points, for which no SMS constraints exist.

the dilepton + MET limits. (There is also a small region of parameter space at low masses where $\tau^{+} \tau^{-}+$MET [77] gives the strongest limit.)

It is important to note here that the constraints on $\tilde{\chi}_{1}^{+} \widetilde{\chi}_{1}^{-} \rightarrow l^{+} l^{-}+$MET actually stem from the $\tilde{l}^{+} \tilde{l}^{-} \rightarrow l^{+} l^{-} \widetilde{\chi}_{1}^{0} \widetilde{\chi}_{1}^{0}$ simplified model (and analogously for $\tau^{+} \tau^{-}+\mathrm{MET}$ ), which has the opposite spin configuration than chargino-pair production followed by chargino decays into sneutrinos. The validity of applying the limits from the slepton searches to the case of chargino-pair production is discussed in appendix A.

Also noteworthy is the fact that most of the excluded points in figures 2 and 3 have some grey points lying below them, which are not excluded or not tested at all. This is corroborated in figure 4, where we present the summary of not tested, allowed and excluded points in the $\tilde{\nu}_{\tau_{1}}$ versus $\tilde{g}$ and $\tilde{\nu}_{\tau_{1}}$ versus $\tilde{\chi}_{1}^{ \pm}$mass planes. In the plots on the left, the excluded points (red) are plotted on on top of the allowed points (blue), while in the plots on the right this plotting order is inverted. As can be seen, only a small part of the parameter space can genuinely be excluded by the SMS results - over most of the regions where the SMS results are valid, there are almost always parameter combinations such that the limits can be avoided. 


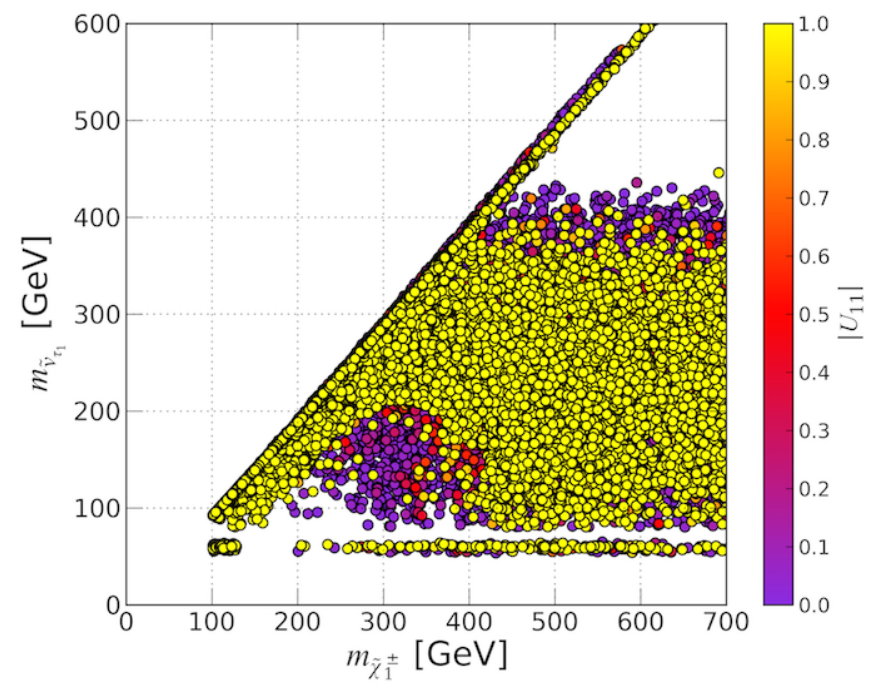

Figure 5. Allowed points in the $\tilde{\nu}_{\tau_{1}}$ vs. $\tilde{\chi}_{1}^{ \pm}$mass plane, with the color code indicating the wino/higgsino content of the $\widetilde{\chi}_{1}^{ \pm}\left(\left|U_{11}\right|=1\right.$ means a pure wino while $\left|U_{11}\right|=0$ means a pure higgsino).

For the dilepton signature originating from chargino-pair production, the chargino mixing plays an important rôle: wino-like charginos have a higher production cross section, and a higher branching fraction into $l \tilde{\nu}_{l 1}$. The limits from $l^{+} l^{-}+$MET searches therefore mostly affect scenarios with wino-like $\widetilde{\chi}_{1}^{ \pm}$, while higgsino scenarios are much less constrained. For illustration see figure 5, which shows the SMS-allowed points in the $\tilde{\nu}_{\tau_{1}}$ versus $\tilde{\chi}_{1}^{ \pm}$mass plane - here the color map gives the size of the $U_{11}$ entry of the chargino mixing matrix, indicating to the wino/higgsino content of the $\widetilde{\chi}_{1}^{ \pm}$. As can be seen, in the region that is in principle constrained by the SMS results the surviving points feature $\widetilde{\chi}_{1}^{ \pm} \mathrm{s}$ that have a large higgsino admixture $\left(\left|U_{11}\right| \lesssim 0.5\right)$. These points have a lower $\widetilde{\chi}_{1}^{+} \widetilde{\chi}_{1}^{-}$production cross section and the $\tilde{\chi}_{1}^{ \pm}$decays preferably into $\tau \tilde{\nu}_{\tau_{1}}$ since the higgsino decay to $e, \mu$ is Yukawa suppressed; $\tau^{+} \tau^{-}+$MET is however a more difficult signature experimentally and thus only constrains a small strip at low $\tilde{\nu}$ mass, cf. the purple points in figure 3 .

Missing topologies. The next question to ask is which are the most important signatures not covered by SMS results. Such information can be used to improve on the interpretation of the LHC searches for new physics. We call these uncovered signatures "missing topologies". For any point passed through SMODELS v1.0.1, we keep up to ten missing topologies sorted by their $\sigma \times \mathcal{B}$. To avoid double counting, missing topologies are evaluated after mass and invisible compressions. The total weight is computed by summing over all diagrams giving the same topology, i.e. ignoring the mass vector of the SUSY states involved. Moreover, $l=e, \mu$ lepton flavors appearing in the final state are summed over (light quark flavors are always summed over). In the following, we only consider MSSM + RN scan points which are not excluded, and we demand that missing topologies have $\sigma \times \mathcal{B} \geq 1 \mathrm{fb}$. The results can be presented in two ways, either by showing the most frequent missing topologies in a certain parameter space, or by selecting for each parameter point the missing topology with the highest cross section. 


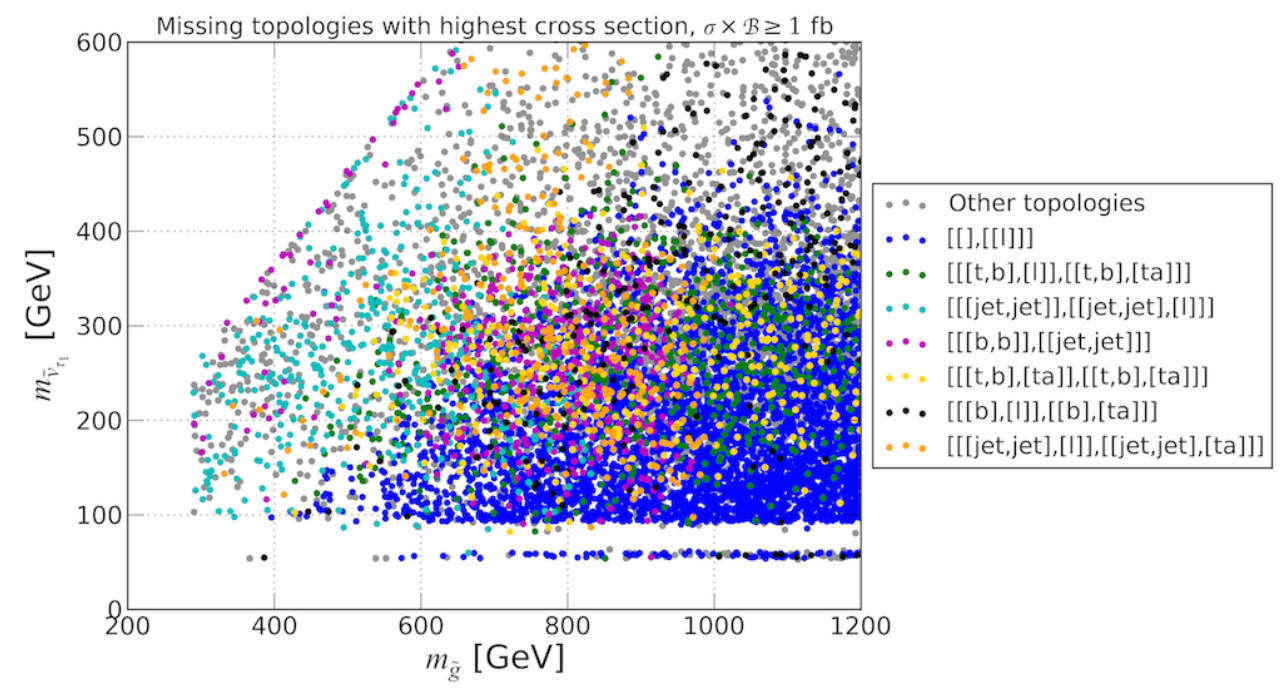

Figure 6. Missing topologies with highest $\sigma \times \mathcal{B}$ in the $\tilde{\nu}_{\tau_{1}}$ vs. $\tilde{g}$ mass plane.

We choose the latter approach to show in figure 6 the missing topologies in the sneutrino- vs. gluino-mass plane. The various processes are denoted in the bracket notation of SMOdeLS, explained in [34]. The structure is [branch1, branch2] for the decay chains ("branches") of the two initially produced SUSY particles; each branch contains inner brackets for each vertex, containing in turn the lists of outgoing standard model particles. Thus, $[[[b, b]],[[j e t, j e t]]]$ denotes gluino-pair production with one gluino decaying into $b \bar{b}+$ MET (via $\tilde{g} \rightarrow b \bar{b} \widetilde{\chi}^{0}, \widetilde{\chi}^{0} \rightarrow \nu \tilde{\nu}$ ) and the other one into $q \bar{q}+$ MET. Likewise, [[ [jet, jet]], [ [jet, jet], [1] ] denotes gluino-pair production with the first gluino decaying via $\tilde{g} \rightarrow q \bar{q} \widetilde{\chi}^{0}, \widetilde{\chi}^{0} \rightarrow \nu \tilde{\nu}$ and the other one via $\tilde{g} \rightarrow q \bar{q}^{\prime} \widetilde{\chi}^{ \pm}, \widetilde{\chi}^{ \pm} \rightarrow l \tilde{\nu} .^{5}$

It is apparent that many points with gluino masses below about $1.2 \mathrm{TeV}$, for which the LHC searches should have good sensitivity, are not excluded by the SMS results because they feature "mixed topologies", where the two pair-produced gluinos undergo different decays (e.g. one gluino decaying into $b \bar{b}$ and the other one into light jets). Since the SMS results for pair-produced sparticles always assume two identical branches, these cases cannot be constrained by SModeLS v1.0.1. Moreover, hadronic final states with additional leptons, as they arise from gluino decays into charginos and the chargino decaying further into a charged lepton $(e, \mu$ or $\tau)$ plus the LSP, do not have any SMS equivalent. Finally, there are no SMS results available for $\tilde{g} \rightarrow t b \widetilde{\chi}_{j}^{ \pm}$, no matter of whether the chargino has any visible decays.

It is also worth noting that over a large part of the parameter space single lepton $+\operatorname{MET}([[],[[1]]]$ in bracket notation) is the most important missing topology. This signature arises from $\widetilde{\chi}_{i}^{0} \widetilde{\chi}_{j}^{ \pm}$production; its importance is corroborated in figure 7 , where one

\footnotetext{
${ }^{5}$ More generically, [[[b,b]], [[jet,jet] $\left.\left.[1]\right]\right]$ denotes production of $X Y$ with $X$ undergoing a 1-step decay chain, $X \rightarrow b \bar{b}+\operatorname{MET}($ branch1=[vertex1] $=[[\mathrm{b}, \mathrm{b}]])$ and $Y$ undergoing a 2-step decay chain, $Y \rightarrow q \bar{q}+Z \rightarrow q \bar{q}+l+\operatorname{MET}($ branch2=[vertex1, vertex2]=[[jet,jet], [1]]); $X$ can be different from $Y$ or both can be the same.
} 


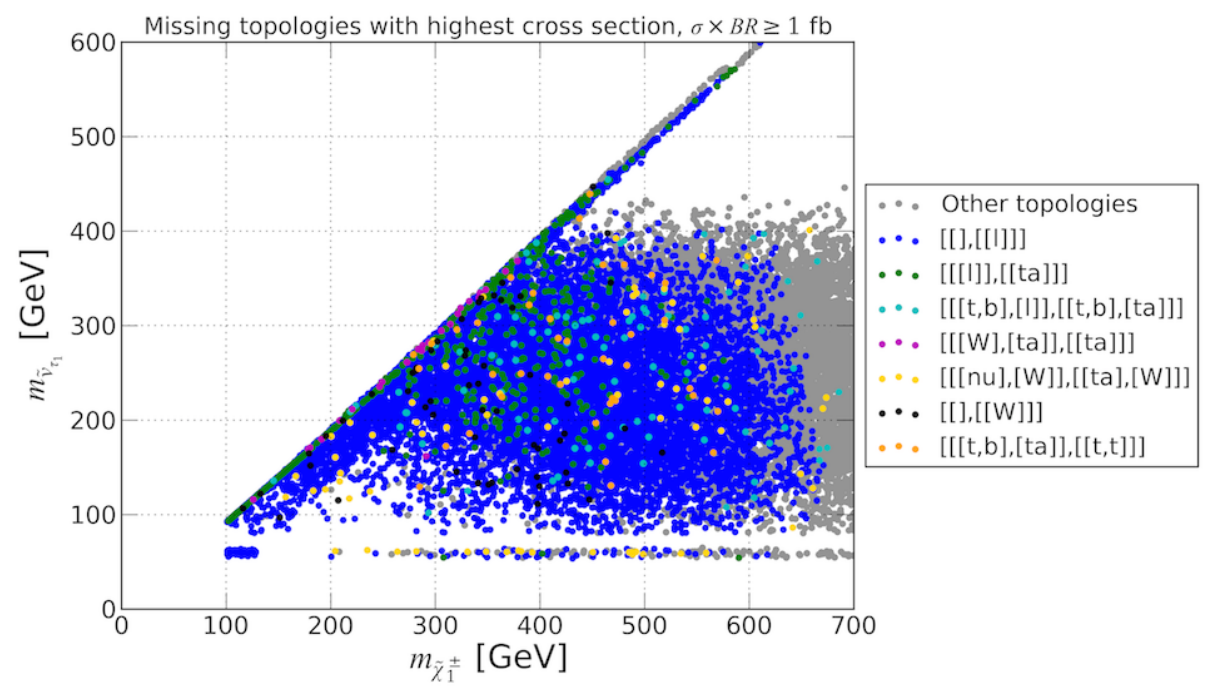

Figure 7. Missing topologies with highest $\sigma \times \mathcal{B}$ in the $\tilde{\nu}_{\tau_{1}}$ vs. $\tilde{\chi}_{1}^{ \pm}$mass plane.

can see that it is indeed dominating the whole sneutrino- vs. chargino-mass plane. (There are also cases where single $W+$ MET is dominant.) The cross section for single lepton + MET production, shown in figure 8, can be very large and should give important additional constraints on the model. While searches for single lepton + MET were performed by both ATLAS [78] and CMS [79], unfortunately no suitable SMS interpretation exists for these analyses. It would be extremely interesting if the experimental collaborations provided upper limit maps and/or efficiency maps for their single lepton + MET analyses in the context of a chargino-sneutrino simplified model.

Having both light EW-inos and light staus can generate decay chains with more 'exotic' signatures, in particular $\widetilde{\chi}_{i}^{ \pm} \widetilde{\chi}_{j}^{0}$ followed by $\widetilde{\chi}_{i}^{ \pm} \rightarrow \nu \tilde{\tau}^{ \pm} \rightarrow \nu W^{ \pm} \tilde{\nu}_{\tau_{1}}$ and $\widetilde{\chi}_{j}^{0} \rightarrow \tau^{ \pm} \tilde{\tau}^{\mp} \rightarrow$ $\tau^{ \pm} W^{\mp} \tilde{\nu}_{\tau_{1}}$. This appears as [[[nu], [W],$[[\mathrm{ta}],[\mathrm{W}]]$ ] (yellow points) in figure 7 and is interesting because the $\widetilde{\chi}_{j}^{0}$ decay produces with the same rate $\tau^{+} W^{-}$and $\tau^{-} W^{+}$: together with the chargino decay this gives rise to a same-sign $W$ signature, $W^{ \pm} W^{ \pm} \tau^{\mp}+$ MET.

Before proceeding it is instructive to take another look at the missing topologies arising from EW-ino and slepton production, but this time ordered by their frequency of occurrence. This is done in figure 9. Not surprisingly we see that besides single lepton ( $e$ or $\mu$ ), single $\tau$ is an important signature. Although it is less clean experimentally, the relative weight of single $e, \mu$ or $\tau+$ MET might potentially give information on the mass pattern of the mostly RH sneutrinos. Another important class of "missing topologies" are different-flavor dileptons ( $[[[1]],[[\mathrm{ta}]]]$ and $\left.\left[[[1]],\left[\left[\mathrm{I}^{\prime}\right]\right]\right]\right)$. Different-flavor dileptons + MET have in principle been considered by ATLAS and CMS in the context of chargino-pair production in the MSSM with the charginos decaying either into $W^{(*)} \widetilde{\chi}_{1}^{0}$ [75] or into $l \nu \widetilde{\chi}_{1}^{0}$ via on-shell sleptons/sneutrinos $[75,76]$. However, the associated SMS limits do not apply to the sneutrino LSP case for various reasons. For example, the leptons from $\tilde{\chi}_{1}^{ \pm} \rightarrow W^{(*)} \widetilde{\chi}_{1}^{0}$ are generally softer than those from $\widetilde{\chi}_{1}^{ \pm} \rightarrow l^{ \pm} \tilde{\nu}_{l}$ decays (for the same $\tilde{\chi}_{1}^{ \pm}$ and LSP masses) because of the additional neutrinos in the $W$ decay. The limits for the 


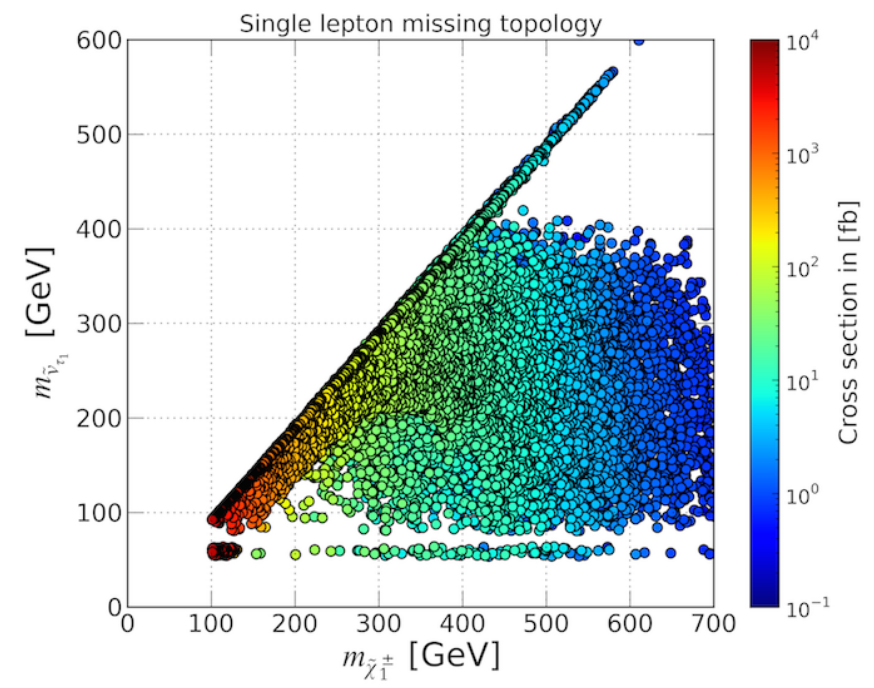

Figure 8. Cross sections $\sigma \times \mathcal{B}$ for the single lepton + MET missing topology for not excluded or not tested points.

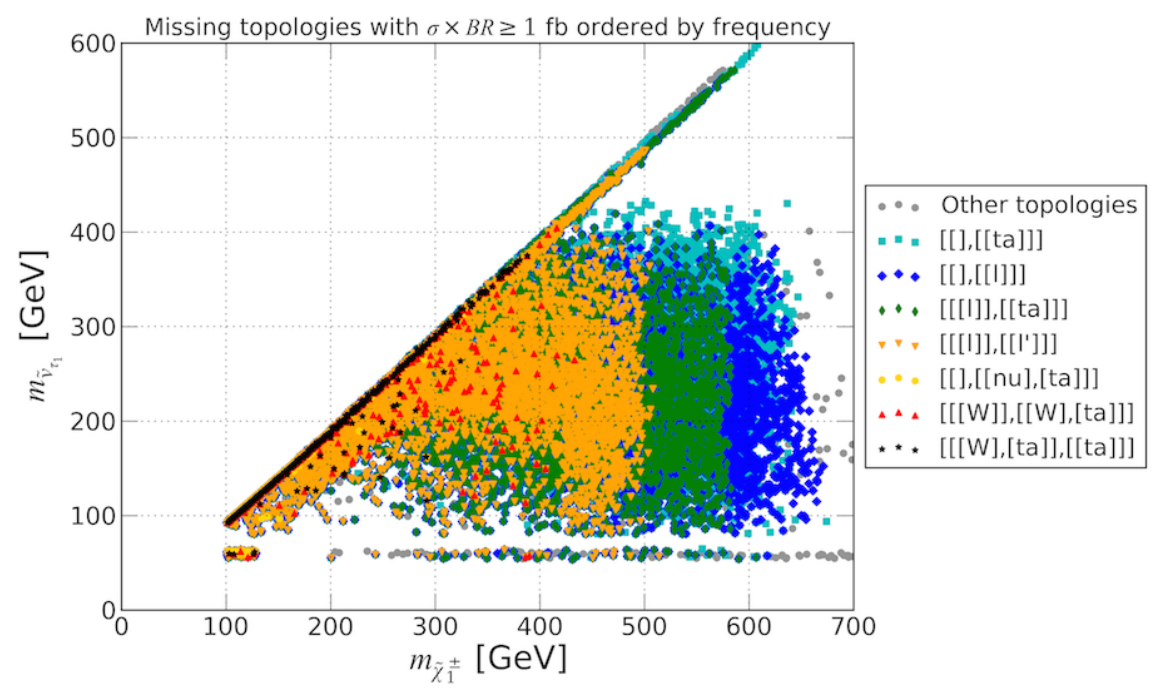

Figure 9. Missing topologies with $\sigma \times \mathcal{B} \geq 1 \mathrm{fb}$ in the sneutrino- vs. chargino-mass plane ordered by frequency of occurrence. The ordering is from top to bottom in the legend, with single tau being the most frequent missing topology, followed by single lepton $(l=e, \mu)$, lepton-tau, and so on. "Other topologies" are shown on top of the legend without considering their total count (however, each single one of them is less frequent than any of the topologies denoted explicitly).

$\tilde{\chi}_{1}^{+} \widetilde{\chi}_{1}^{-} \rightarrow 2 \times \tilde{l} \nu($ or $\tilde{\nu} l) \rightarrow 2 \times l \nu \widetilde{\chi}_{1}^{0}$ simplified model are also not applicable because they involve an additional intermediate mass scale.

Finally, the $[[\mathrm{W}]],[[\mathrm{W}],[\mathrm{ta}]]]$ topology again gives rise to same-sign $W$ 's, see the red triangles in figure 9. Similarly it is possible to have same sign $\tau$ 's arising from $[[\mathrm{W}],[\mathrm{ta}]],[\mathrm{ta}]]]$ (black stars). In this case, after $\widetilde{\chi}_{i}^{0} \widetilde{\chi}_{j}^{ \pm}$production, the decay chain is $\tilde{\chi}_{i}^{0} \rightarrow W^{\mp} \tilde{\chi}_{k}^{ \pm} \rightarrow W^{\mp} \tau^{ \pm} \tilde{\nu}_{\tau_{1}}$ and $\tilde{\chi}_{j}^{ \pm} \rightarrow \tau^{ \pm} \tilde{\nu}_{\tau_{1}}$. 

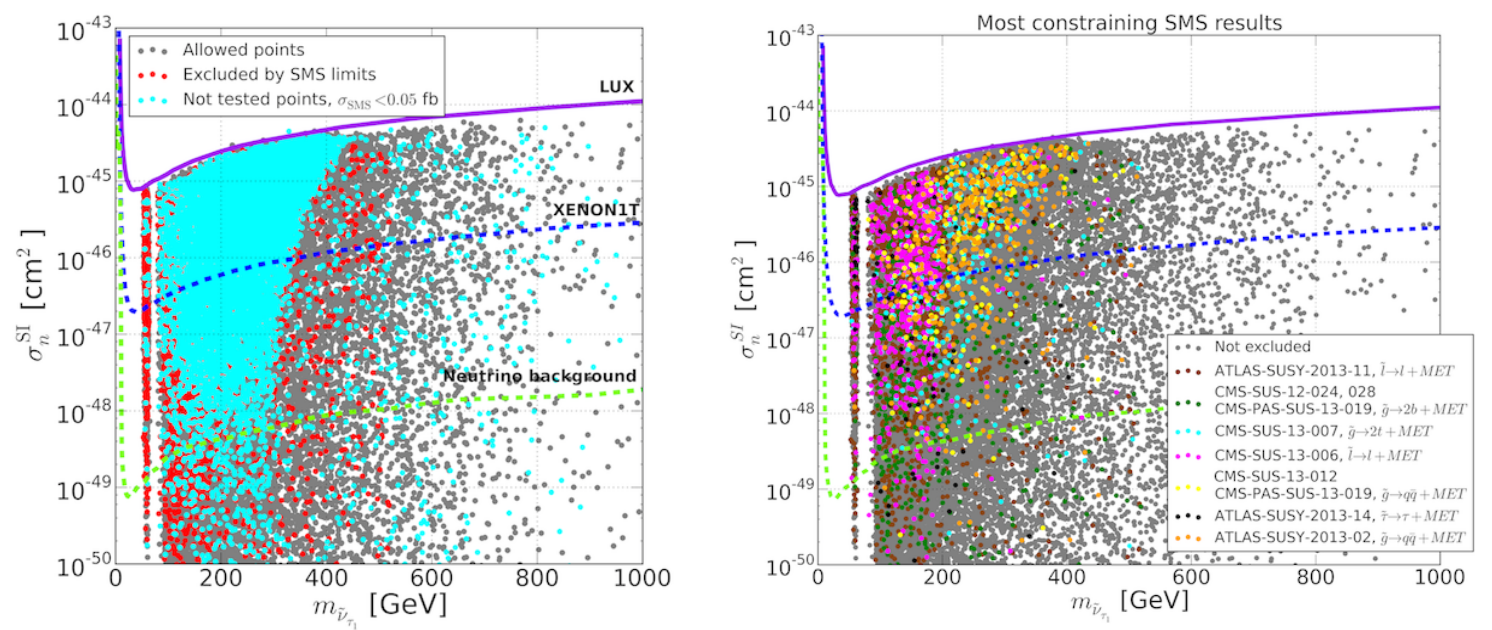

Figure 10. Complementarity of LHC and direct DM detection experiments. The panel on the left shows SMS allowed, excluded and not tested points in the plane of $\sigma_{n}^{\text {SI }}$ vs. $m_{\tilde{\nu}_{\tau_{1}}}$. The panel on the right shows the breakdown of most constraining analyses for the points that are excluded by the SMS limits (for the sake of comparison, the allowed points are shown in grey). In both panels, the solid magenta lines and the dashed blue lines are the current exclusion limit by LUX and the forecasted sensitivity of XENON1T experiment respectively, while the dashed light green line corresponds to the predicted neutrino coherent scattering on nuclei.

Complementarity with direct DM searches. Let us finally turn to the complementarity of LHC and direct DM searches - recall that all points in our scans are consistent with DM constraints, as described in table 1. In figure 10, left panel, we plot the allowed (gray), excluded (red) and not tested points (cyan) as a function of the sneutrino mass and the SI scattering cross section. In the same plot we also show the forecasted sensitivity of XENON1T after two years of scientific run [80] and the predicted value for neutrino coherent scattering on nuclei [81], which can be an irreducible background for direct detection experiments. From this plot, the complementarity between the two type of searches is striking. Points with a SI elastic cross section well below the neutrino background, and hence not detectable by direct detection experiments, are already excluded by SMS results. On the other hand, a bulk of points allowed (or even more interestingly, not tested) by SMS results is well in the reach of XENON1T, expected to start running in 2015. Notice however that there still exist combinations of parameters that allow sneutrino DM to escape both direct detection and LHC searches, represented by the cyan points below the neutrino background curve. In the MSSM+RN, DM direct searches are basically sensitive to the mass of the LSP and its couplings with the Higgs and $Z$ bosons. The rest of the SUSY mass spectrum is not relevant. This is different with respect to the MSSM with the neutralino LSP, where the interaction with the quarks is mediated as well by squarks on $t$-channel. This is clearly visible in the right panel of figure 10, which shows the most constraining SMS analyses. In figures 2 and 3 these SMS analyses are typically correlated with the gluino or chargino mass, while now they are scattered all over the $\sigma_{n}^{\text {SI }}$ versus $m_{\tilde{\nu}_{\tau_{1}}}$ plane. 

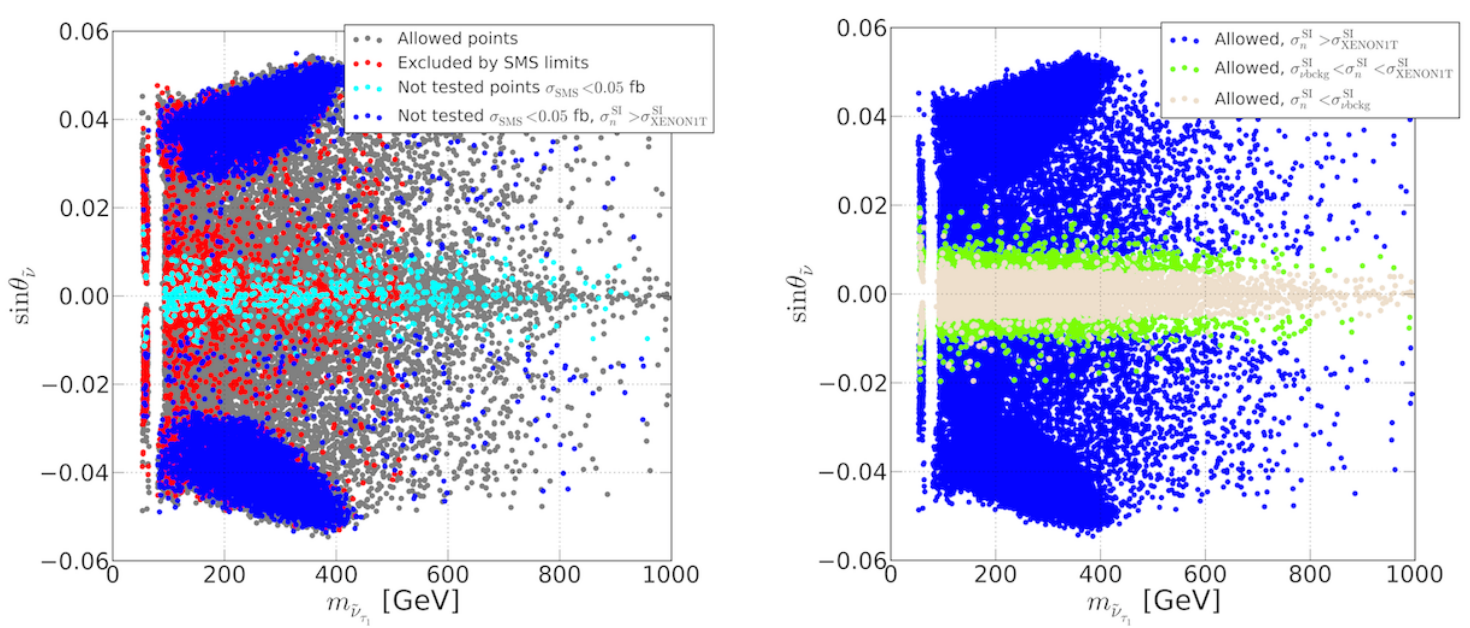

Figure 11. On the left allowed (grey), excluded (red) and not tested (blue and cyan) points are shown in the plane of sneutrino mass versus mixing angle. The subset of points with exceedingly small $\sigma \times \mathcal{B}$ at the LHC but in the reach of XENON1T is visualised in blue. On the right, we show the SMS allowed points in the sneutrino mass versus mixing angle plane, subdivided in blue points, which are in the reach of XENON1T and in light green (light grey) points, which are above (below) the neutrino background.

The same set of allowed, excluded and not tested points are plotted as a function of the sneutrino mixing angle in the left panel of figure 11. The bulk of not tested points in the reach of XENON1T (dark blue points) has, as expected, relatively large mixing angles, corresponding to sizeable contributions from $Z$ boson exchange to the SI scattering cross section. Excluded red points are scattered everywhere in the $\sin \theta_{\tilde{\nu}}$ vs. $m_{\tilde{\nu}_{\tau_{1}}}$ plane and probe also very RH sneutrinos. In the right panel of figure 11 we see that among the allowed points, XENON1T can constrain a large portion of the sneutrino parameter space, while the very RH sneutrinos will remain inaccessible to future direct detection detectors. In general the points with negligible mixing angles have $\tilde{\nu}_{\tau_{1}}$ as LSP and the neutralino as NLSP, which tends to be almost degenerate with chargino. The relic density is then actually achieved by co-annihilation of neutralino-chargino and then communicated to the mostly sterile LSP (see [27] for details). Such scenarios are very difficult to test.

\section{Conclusions}

Scenarios with a sneutrino as the LSP are an interesting alternative to MSSM models with neutralino LSPs. Indeed in SUSY models with a RH neutrino superfield (MSSM+RN) the fermionic field contributes to neutrino masses while the scalar field contributes to the DM candidate, which is a mixed, however mostly $\mathrm{RH}$, sneutrino.

The collider phenomenology of the MSSM+RN can be quite different from the typical MSSM case. It is therefore interesting and relevant to ask how the SUSY search results from Run 1 of the LHC, which were mostly designed with the MSSM in mind, constrain sneutrino LSP scenarios. To address this question, we used SModeLS v1.0.1 for testing the 
MSSM+RN against more than 60 results from CMS and ATLAS searches in the context of so-called Simplified Model Spectra (SMS). More precisely, by considering the model parameter space where the sneutrino is a good DM candidate compatible with all current constraints, we assessed 1.) the constraining power of the current SMS results on such scenarios and 2.) the most relevant signatures not covered by the SMS approach.

Concerning point 1.), we found that the dilepton + MET searches are among the most relevant ones, constraining sneutrino masses up to about $210 \mathrm{GeV}$ and mostly wino-like charginos up to $m_{\widetilde{\chi}_{1}^{ \pm}} \approx 440 \mathrm{GeV}$. It is important to note here that this amounts to reinterpreting the ATLAS and CMS searches for $p p \rightarrow \tilde{l}^{+} \tilde{l}^{-} \rightarrow l^{+} l^{-} \widetilde{\chi}_{1}^{0} \widetilde{\chi}_{1}^{0}$ in terms of $p p \rightarrow$ $\tilde{\chi}_{1}^{+} \widetilde{\chi}_{1}^{-} \rightarrow l^{+} l^{-} \tilde{\nu}_{l} \tilde{\nu}_{l}$ (the validity of this is discussed in appendix A). Hadronic SUSY searches exclude gluinos masses up to $m_{\tilde{g}} \approx 1200 \mathrm{GeV}$ and LSP masses up to $m_{\tilde{\nu}_{1}} \approx 500 \mathrm{GeV}$. Nonetheless in general we find that only a very limited portion of the parameter space can be properly excluded by SMS results. For most points in the $\left(m_{\tilde{g}}, m_{\tilde{\nu}_{1}}\right)$ or $\left(m_{\tilde{\chi}_{1}^{ \pm}}, m_{\tilde{\nu}_{1}}\right)$ planes there exist parameter combinations that allow to avoid all limits. Indeed, most of the parameter space is either allowed (SMS constraints exist for the specific topologies of the point but all $\sigma \times \mathcal{B}$ of these topologies are below their 95\% CL upper limits) or not tested at all (there are no existing SMS constraints for the specific topologies of the point or each topology has a $\sigma \times \mathcal{B}$ which is smaller than 1 event at LHC Run 1). Direct DM searches are complementary to the SMS constraints: many points that are not tested by SMS results can potentially be excluded by XENON1T. Vice versa, points well below the neutrino background, hence not reachable by future DM detectors, are already excluded by SMS results.

The second main result of this paper concerns point 2.), i.e. the study of the allowed points in terms of missing topologies. In the hadronic sector, pair-produced gluinos with masses well in the reach of LHC Run 1 are not constrained because they feature one or more of the following:

- additional leptons: since the gluino cannot directly decay into the sneutrino LSP, the hadronic final state is often accompanied by leptons;

- mixed topologies: each of the pair-produced gluinos undergoes a different decay;

- the gluinos decay into $t b$ final states.

None of these possibilities are covered by the current SMS results. Note here that the last two items are also common in the MSSM, as described in [34]. For EW production, missing topologies include:

- single leptons;

- single $W \mathrm{~s}$;

- different-flavour opposite-sign leptons;

- same-sign $W$ 's or same-sign taus (accompanied respectively by additional leptons/ taus, or $W \mathrm{~s}$ ). 
While such signatures have been searched for by the SUSY and/or exotics groups in ATLAS and CMS, the results do not exist in terms of appropriate SMS interpretations. Such an SMS interpretation would be very interesting in particular for the mono-lepton + MET case, which promises to have a considerable impact for constraining the MSSM+RN model. ${ }^{6}$

A final comment is in order. While the SMS approach is very convenient for the characterisation of new physics signatures and vast surveys of parameter spaces, it clearly has its limitations. Given the high interest in non-standard SUSY (and other new physics) scenarios, we urge the experimental collaborations to document their analyses in a way that they can conveniently be re-casted in public simulation frameworks like CHECKMATE [82] or the MAdANALysis 5 PAD [83]. (See also the recommendations in [83] and [84] in this context). This would allow to go beyond the limitations of SMS approach and give a much more rigorous assessment of the constraints in a large variety of new physics models, including the sneutrino DM scenario discussed in this paper. Unfortunately we are still a long way from this.

\section{Acknowledgments}

SK, SuK, and UL thank their colleagues from the SModeLS developer team, Andre Lessa, Veronica and Wolfgang Magerl, Michael Traub and Wolfgang Waltenberger for many helpful discussions. We also thank B. Dumont, B. Fuks, E. Conte and D. Sengupta for helpful discussions on recasting with MADANALYsis 5. SuK moreover acknowledges discussions with R. Schoeffbeck.

This work was supported in part by the ANR project DMAstroLHC and the "Investissements d'avenir, Labex ENIGMASS". CA is supported by the ERC project 267117 hosted by UPMC-Paris 6, PI J. Silk. MECC is supported by Fundação de Amparo à Pesquisa do Estado de São Paulo (FAPESP). SuK is supported by the "New Frontiers" program of the Austrian Academy of Sciences. UL is supported by the Labex ENIGMASS; she also gratefully acknowledges the hospitality of LPSC Grenoble during research visits prior to starting her $\mathrm{PhD}$ thesis at the LPSC.

\section{A Validity of slepton search results for chargino-pair production with decay into lepton+sneutrino}

In the spirit of the SMOdELS v1.0.1 philosophy, we apply SMS constraints for the $l^{+} l^{-}+$ MET topology, obtained in the context of pair production of charged sleptons, $p p \rightarrow$ $\tilde{l}^{+} \tilde{l}^{-}$followed by $\tilde{l}^{ \pm} \rightarrow l^{ \pm} \tilde{\chi}_{1}^{0}$ to the case of chargino-pair production $p p \rightarrow \tilde{\chi}_{1}^{+} \tilde{\chi}_{1}^{-}$followed by $\tilde{\chi}_{1}^{ \pm} \rightarrow l^{ \pm} \tilde{\nu}_{l 1}$, despite the opposite spin configuration. This can only be valid if the signal selection efficiencies in both scenarios are comparable. To test this assumption, we

\footnotetext{
${ }^{6}$ This could be done analogous to the existing $\widetilde{\chi}_{1}^{ \pm} \widetilde{\chi}_{2}^{0}\left(\widetilde{\chi}_{1}^{ \pm} \rightarrow W^{ \pm} \widetilde{\chi}_{1}^{0}, \widetilde{\chi}_{2}^{0} \rightarrow Z^{0} \widetilde{\chi}_{1}^{0}\right)$ simplified models that are already assessed by the ATLAS and CMS SUSY groups, but with the chargino decaying to $100 \%$ into $l^{ \pm} \tilde{\nu}_{l}$ and the neutralino decaying $100 \%$ into $\nu \tilde{\nu}_{l}$. However, since the chargino and neutralino masses need not be degenerate, we propose to consider as a first step $\tilde{\chi}_{1}^{ \pm} \widetilde{\chi}_{1}^{0}$ production followed by $\tilde{\chi}_{1}^{ \pm} \rightarrow l^{ \pm} \tilde{\nu}_{l}$ and $\tilde{\chi}_{1}^{0} \rightarrow \nu \tilde{\nu}_{l}$. The cross section upper limits should be provided in the chargino- versus sneutrino mass plane for different neutralino masses, for the cases $l=e, \mu$ and $l=\tau$, and if computationally feasible also for $l=e, \mu, \tau$ assuming equal rates.
} 

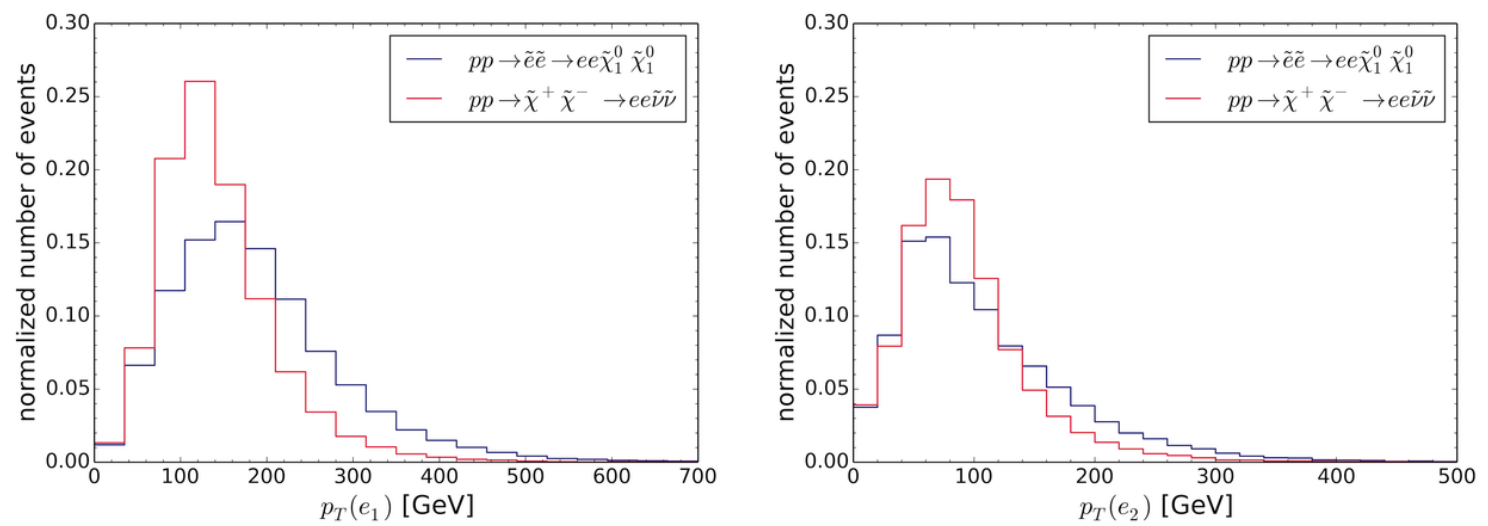

Figure 12. Comparison of the $p_{T}$ distributions of electrons originating from selectron decays in the MSSM and from chargino decays in MSSM+RN, at the level of reconstructed events. The benchmark scenarios used are $\left(m_{\tilde{l}^{ \pm}}, m_{\tilde{\chi}_{1}^{0}}\right)=(270,100) \mathrm{GeV}$ for the MSSM case and $\left(m_{\tilde{\chi}_{1}^{ \pm}}, m_{\tilde{\nu}_{1}}\right)=$ $(270,100) \mathrm{GeV}$ for the MSSM+RN case. See text for details.

use the recast code [85] for ATLAS search in final states with two leptons and missing transverse momentum, ATLAS-SUSY-2013-11 [75], which is available in the framework of the MadAnalysis 5 "Public Analysis Database" [83]. We consider two benchmark scenarios in the simplified-model spirit, an MSSM one with $\left(m_{\tilde{l}^{ \pm}}, m_{\tilde{\chi}_{1}^{0}}\right)=(270,100) \mathrm{GeV}$ and an MSSM+RN one with $\left(m_{\tilde{\chi}_{1}^{ \pm}}, m_{\tilde{\nu}_{1}}\right)=(270,100) \mathrm{GeV}$. Events are generated with MadGraph 5 [86, 87] and Pythia 6.4 [57] and then passed through Delphes 3 [88] for the simulation of the detector effects. ${ }^{7}$ For simplicity, in the following we restrict our study to pair-production of selectrons for the MSSM case, and pair-production of charginos decaying exclusively via electrons in the MSSM+RN case.

The event selection requires two opposite sign (OS), same flavor (SF) leptons with high transverse momentum, concretely $p_{T}>35 \mathrm{GeV}$ and $p_{T}>20 \mathrm{GeV} .{ }^{8}$ Figure 12 compares the $p_{T}$ distributions in the two benchmark scenarios, in the left panel for the harder electron, $e_{1}$, in the right panel for the second electron, $e_{2}$. The bin sizes are chosen such that the first bin corresponds to the events that do not pass the $p_{T}>35 \mathrm{GeV}$ (left panel) or $p_{T}>$ $20 \mathrm{GeV}$ (right panel) requirement. We see that the electrons originating from selectron-pair production tend to be harder than those originating from chargino-pair production.

The analysis further requires the invariant mass of the lepton pair to be outside the $Z$ window, and $\tau$ s and jets are vetoed. Finally, three signal regions are defined by thresholds on the $m_{T 2}$ ("stransverse mass") variable $[89,90]$ that is used for reducing the $t \bar{t}$ and $W t$ backgrounds: $m_{T 2}>90,>120$ and $>150 \mathrm{GeV}$. The $m_{T 2}$ distributions after the preselection cuts are shown in figure 13. It can be seen that the distributions intersect around the minimum required value of $m_{T 2}=90 \mathrm{GeV}$; events with electrons originating from chargino decays are more likely to pass this cut.

\footnotetext{
${ }^{7}$ Note that for the reconstruction of events with a sneutrino LSP it is necessary to define the sneutrino as MET, by adding a corresponding EnergyFraction entry in the DeLPHES card.

${ }^{8}$ We consider here only the part of the analysis that is relevant for the SMS result used to constrain the sneutrino LSP scenario in section 4.
} 


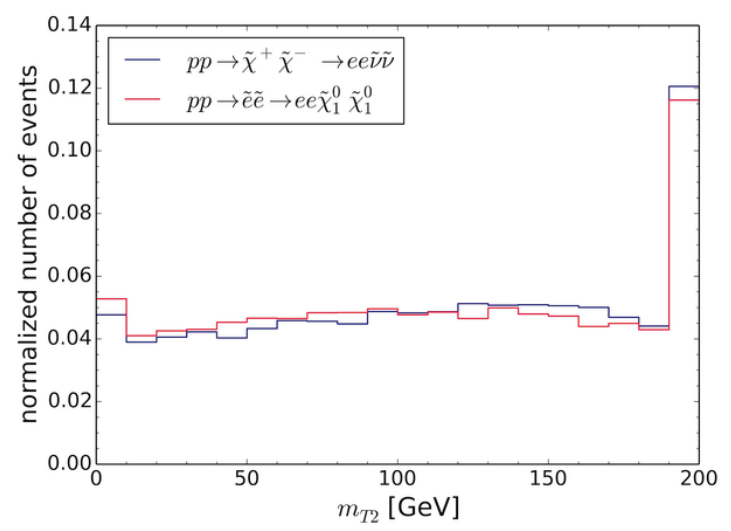

Figure 13. Comparison of the $m_{T 2}$ distributions for the two benchmark scenarios after all preselection cuts.

To see the net effect on the signal efficiencies, table 2 shows the complete cut-flow comparison for the two benchmark scenarios. As expected, differences arise in the first cut, selecting high $p_{T}$ OS lepton pairs, and when applying the lower bounds for $m_{T 2}$. Because of the softer $p_{T}$ distribution in case of chargino production+decay, there are fewer events passing the first cut for this scenario. However, the opposite is true for the $m_{T 2}$ cut. Ultimately, the efficiencies are comparable in all signal regions, and even somewhat higher for the MSSM+RN scenario.

To check that this is still true closer to the kinematic edge, we reproduce the cut-flows for a second set of benchmark scenarios with an LSP mass of $200 \mathrm{GeV}$. As can be seen in table 3, we find a similar behaviour in this case. We conclude that we can safely apply the SMS upper limits given by the experimental collaborations in the context of slepton-pair production in the MSSM to constrain chargino-pair production followed by decays into $l \tilde{\nu}_{l}$ in the MSSM+RN.

\section{B Lifetimes of long-lived particles}

As mentioned in section 3.2, a considerable number of the scan points comprise long-lived sparticles. These occur mostly when enforcing light gluinos or squarks; in this case about $30 \%$ of the points feature long-lived particles, while the fraction is below $1 \%$ without this constraint. The long-lived particles are predominantly gluinos $(85 \%)$, mostly in the case where it is the NLSP, and in a few points where $\tilde{\chi}_{1}^{0}$ is slightly (up to about $50 \mathrm{GeV}$ ) lighter than the gluino. Apart from that we find points with long-lived stops or staus in case they are the NLSP, as well as single points with long-lived charginos. Here we will focus on the long-lived gluinos and stops, long-lived staus have been discussed before in [27].

In the MSSM long-lived gluinos appear when all squarks are extremely heavy, e.g. in split-SUSY scenarios. In case of the MSSM+RN with a sneutrino LSP additional causes come into play. If the gluino is the NLSP, its decay will proceed only via virtual squarks and gauginos, yielding an effective four body decay, $\tilde{g} \rightarrow q q \nu \tilde{\nu}$ (virtual $\tilde{q}$ and $\tilde{\chi}^{0}$ ) or $\tilde{g} \rightarrow q q^{\prime} l \tilde{\nu}$ (virtual $\tilde{q}$ and $\tilde{\chi}^{ \pm}$). The gluino lifetime will therefore depend not only on the squark mass, 


\begin{tabular}{|l|c|c|}
\hline Cut & Slepton production & Chargino production \\
\hline \multicolumn{2}{|c|}{ Common preselection } \\
\hline Initial number of events & 50000 & 50000 \\
2 OS leptons & 35133 & 33464 \\
$m_{l l}>20 \mathrm{GeV}$ & 35038 & 33337 \\
$\tau$ veto & 35007 & 33318 \\
ee leptons & 35007 & 33318 \\
jet veto & 20176 & 19942 \\
$Z$ veto & 19380 & 18984 \\
\hline \multicolumn{2}{|c|}{} & 11346 \\
\hline$m_{T 2}>90 \mathrm{GeV}$ & 8520 & 8828 \\
$m_{T 2}>120 \mathrm{GeV}$ & 5723 & 5926 \\
$m_{T 2}>150 \mathrm{GeV}$ & Different $m_{T 2}$ regions \\
\hline
\end{tabular}

Table 2. Comparison of the cut-flows for $p p \rightarrow \tilde{e} \tilde{e} \rightarrow e^{+} e^{-} \widetilde{\chi}_{1}^{0} \widetilde{\chi}_{1}^{0}$ and $p p \rightarrow \widetilde{\chi}_{1}^{+} \widetilde{\chi}_{1}^{-} \rightarrow e^{+} e^{-} \tilde{\nu}_{1} \tilde{\nu}_{1}$ with $\left(m_{\tilde{l}^{ \pm}}, m_{\tilde{\chi}_{1}^{0}}\right)=(270,100) \mathrm{GeV}$ and $\left(m_{\tilde{\chi}_{1}^{ \pm}}, m_{\tilde{\nu}_{1}}\right)=(270,100) \mathrm{GeV}$, respectively.

\begin{tabular}{|l|c|c|}
\hline Cut & Slepton production & Chargino production \\
\hline \multicolumn{2}{|c|}{ Common preselection } \\
\hline Initial number of events & 50000 & 50000 \\
2 OS leptons & 29291 & 27244 \\
$m_{l l}>20 \mathrm{GeV}$ & 29082 & 26964 \\
$\tau$ veto & 29050 & 26956 \\
ee leptons & 29050 & 26956 \\
jet veto & 16834 & 16114 \\
$Z$ veto & 15281 & 14025 \\
\hline \multicolumn{2}{|c|}{} & 3028 \\
\hline$m_{T 2}>90 \mathrm{GeV}$ & 85 & 3198 \\
$m_{T 2}>120 \mathrm{GeV}$ & 0 & 140 \\
$m_{T 2}>150 \mathrm{GeV}$ & Different $m_{T 2}$ regions \\
\hline
\end{tabular}

Table 3. As table 2 but for $\left(m_{\tilde{l}^{ \pm}}, m_{\tilde{\chi}_{1}^{0}}\right)=(270,200) \mathrm{GeV}$ and $\left(m_{\tilde{\chi}_{1}^{ \pm}}, m_{\tilde{\nu}_{1}}\right)=(270,200) \mathrm{GeV}$.

but also on the gaugino masses and mixings, as well as the sneutrino mixing angle. Metastable gluinos can thus appear even if the squarks are not completely decoupled. The gluino lifetime as a function of its mass is shown in figure 14. The left plot illustrates the depencence on the sneutrino mass, the right plot the dependence on the sneutrino mixing. We can distinguish two general regions. First, we observe an exponential dependence of 

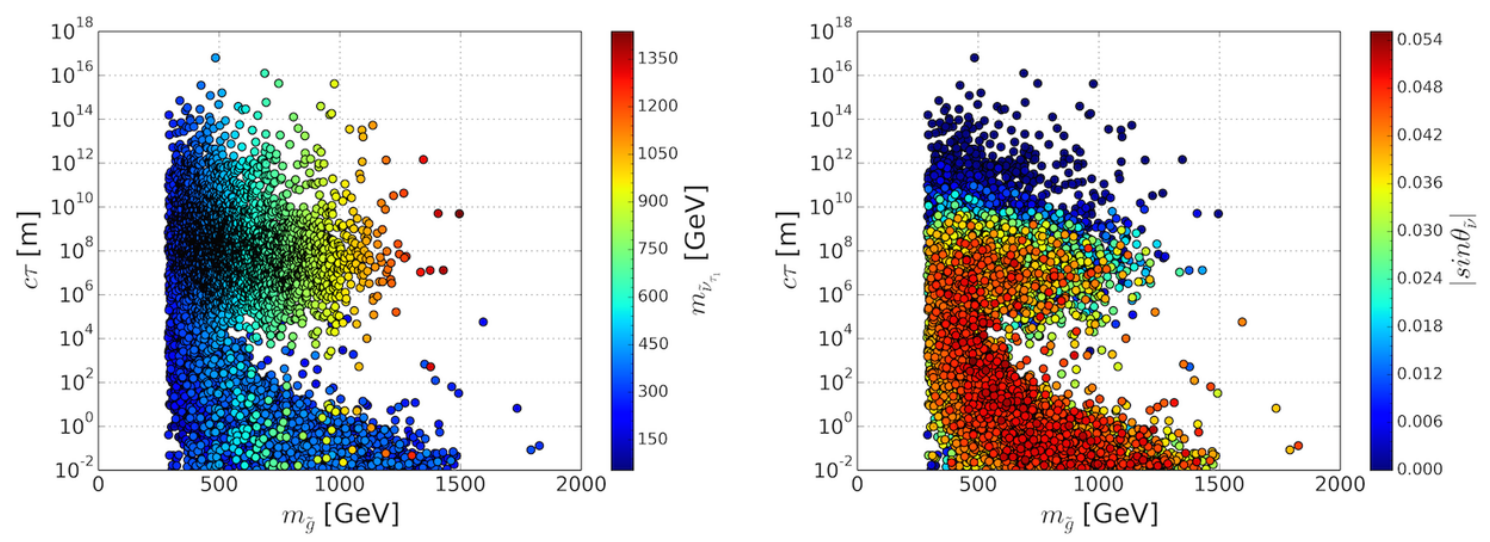

Figure 14. Lifetimes $c \tau$ in $[\mathrm{m}]$ for long-lived gluinos, the color code indicates the LSP mass (left) and the sneutrino mixing angle (right).
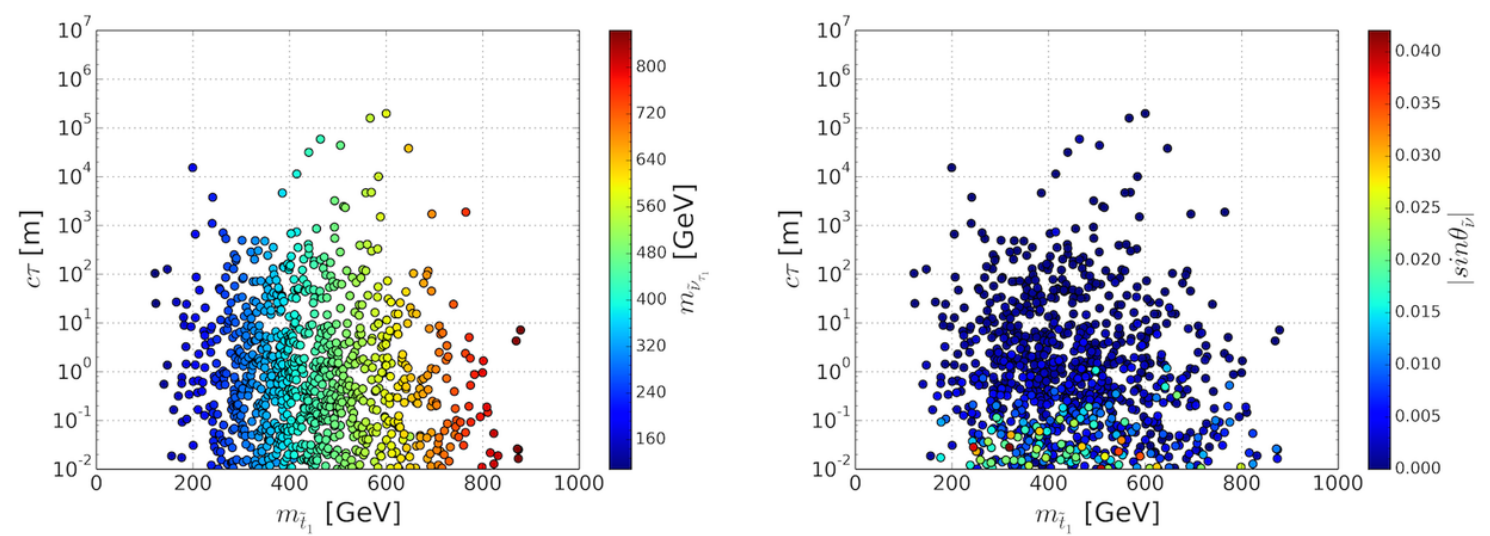

Figure 15. Lifetimes $c \tau$ in $[\mathrm{m}]$ for long-lived stops, the color code indicates the LSP mass (left) and the sneutrino mixing angle (right).

the lifetime on the gluino mass for decay lengths of $10 \mathrm{~mm}$ up to $10^{4} \mathrm{~m}$. Here the lifetime is largely independent of the sneutrino mass. Moreover lifetimes at constant gluino masses are longer for heavier squarks and gauginos. In this region we generally find large mixing angles $\sin \theta_{\tilde{\nu}}$, but heavy gauginos and squarks. Points with very small mixing angles may also appear in this region, in the case that the mass of the lightest neutralino is below the gluino mass. The second region, with lifetimes longer than $10^{4} \mathrm{~m}$, and up to $10^{17} \mathrm{~m}$, shows a very different behaviour. We can see a clear correlation between gluino and sneutrino masses in this region, with longer lifetimes found for smaller mass splittings. The lifetimes moreover increase when going to very small sneutrino mixing angles, with the maximum lifetimes achieved for $\sin \theta_{\tilde{\nu}}$ going to zero.

Likewise, if the stop is the NLSP ${ }^{9}$ and has a small mass difference with the sneutrino, it can be long-lived, see figure 15. As seen for the gluinos, the lifetime depends strongly on the sneutrino mixing.

\footnotetext{
${ }^{9}$ If the stop mass is close to the gluino mass, both stop and gluino may be long-lived.
} 
Both long lived gluinos and long lived stops can be constrained by searches for Rhadrons, see [91, 92] for R-hadrons escaping the detector, [93] for stopped R-hadrons, or [94] for metastable gluinos decaying in flight inside the detector. However, large uncertainties arise from modeling both the hadronisation and the strong interaction of the R-hadron with the detector. Therefore no collider constraints on long-lived sparticles have been included.

Additionally, cosmological constraints become important for gluino lifetimes of about $100 \mathrm{~s}$ $\left(10^{10} \mathrm{~m}\right)$ [95]. Lifetimes of that order would affect the fraction of heavy nuclei produced during the Big Bang nucleosynthesis. Longer lifetimes can further be constrained by searches for diffuse gamma ray background, distortions in the CMBR and heavy isotopes.

Open Access. This article is distributed under the terms of the Creative Commons Attribution License (CC-BY 4.0), which permits any use, distribution and reproduction in any medium, provided the original author(s) and source are credited.

\section{References}

[1] ATLAS collaboration, Observation of a new particle in the search for the Standard Model Higgs boson with the ATLAS detector at the LHC, Phys. Lett. B 716 (2012) 1 [arXiv: 1207.7214] [INSPIRE].

[2] CMS collaboration, Observation of a new boson at a mass of $125 \mathrm{GeV}$ with the CMS experiment at the LHC, Phys. Lett. B 716 (2012) 30 [arXiv:1207.7235] [INSPIRE].

[3] https://twiki.cern.ch/twiki/bin/view/AtlasPublic/SupersymmetryPublicResults.

[4] https://twiki.cern.ch/twiki/bin/view/CMSPublic/PhysicsResultsSUS.

[5] A. Djouadi and J. Quevillon, The MSSM Higgs sector at a high $M_{S U S Y}$ : reopening the low $\tan \beta$ regime and heavy Higgs searches, JHEP 10 (2013) 028 [arXiv:1304.1787] [InSPIRE].

[6] G.F. Giudice and A. Romanino, Split supersymmetry, Nucl. Phys. B 699 (2004) 65 [hep-ph/0406088] [INSPIRE].

[7] G. Bhattacharyya and T.S. Ray, Naturally split supersymmetry, JHEP 05 (2012) 022 [arXiv: 1201.1131] [INSPIRE].

[8] K. Benakli, L. Darmé, M.D. Goodsell and P. Slavich, A Fake Split Supersymmetry Model for the 126 GeV Higgs, JHEP 05 (2014) 113 [arXiv: 1312.5220] [INSPIRE].

[9] L.J. Hall and Y. Nomura, Spread Supersymmetry, JHEP 01 (2012) 082 [arXiv:1111.4519] [INSPIRE].

[10] H.K. Dreiner, M. Krämer and J. Tattersall, How low can SUSY go? Matching, monojets and compressed spectra, Europhys. Lett. 99 (2012) 61001 [arXiv:1207.1613] [INSPIRE].

[11] J. Fan, M. Reece and J.T. Ruderman, Stealth Supersymmetry, JHEP 11 (2011) 012 [arXiv: 1105.5135] [INSPIRE].

[12] F. Borzumati and Y. Nomura, Low scale seesaw mechanisms for light neutrinos, Phys. Rev. D 64 (2001) 053005 [hep-ph/0007018] [INSPIRE].

[13] N. Arkani-Hamed, L.J. Hall, H. Murayama, D. Tucker-Smith and N. Weiner, Small neutrino masses from supersymmetry breaking, Phys. Rev. D 64 (2001) 115011 [hep-ph/0006312] [INSPIRE]. 
[14] WMAP collaboration, G. Hinshaw et al., Nine-Year Wilkinson Microwave Anisotropy Probe (WMAP) Observations: Cosmological Parameter Results, Astrophys. J. Suppl. 208 (2013) 19 [arXiv: 1212.5226] [INSPIRE].

[15] Planck collaboration, P.A.R. Ade et al., Planck 2013 results. XVI. Cosmological parameters, Astron. Astrophys. 571 (2014) A16 [arXiv:1303.5076] [inSPIRE].

[16] T. Asaka, K. Ishiwata and T. Moroi, Right-handed sneutrino as cold dark matter, Phys. Rev. D 73 (2006) 051301 [hep-ph/0512118] [INSPIRE].

[17] F. Deppisch and A. Pilaftsis, Thermal Right-Handed Sneutrino Dark Matter in the F(D)-Term Model of Hybrid Inflation, JHEP 10 (2008) 080 [arXiv:0808.0490] [INSPIRE].

[18] D.G. Cerdeno and O. Seto, Right-handed sneutrino dark matter in the NMSSM, JCAP 08 (2009) 032 [arXiv:0903.4677] [INSPIRE].

[19] S. Khalil, H. Okada and T. Toma, Right-handed Sneutrino Dark Matter in Supersymmetric B-L Model, JHEP 07 (2011) 026 [arXiv: 1102.4249] [INSPIRE].

[20] K.-Y. Choi and O. Seto, A Dirac right-handed sneutrino dark matter and its signature in the gamma-ray lines, Phys. Rev. D 86 (2012) 043515 [arXiv: 1205.3276] [INSPIRE].

[21] G. Bélanger, M. Kakizaki, E.K. Park, S. Kraml and A. Pukhov, Light mixed sneutrinos as thermal dark matter, JCAP 11 (2010) 017 [arXiv: 1008.0580] [INSPIRE].

[22] B. Dumont, G. Bélanger, S. Fichet, S. Kraml and T. Schwetz, Mixed sneutrino dark matter in light of the 2011 XENON and LHC results, JCAP 09 (2012) 013 [arXiv:1206.1521] [INSPIRE].

[23] D. Hooper, J. March-Russell and S.M. West, Asymmetric sneutrino dark matter and the Omega(b)/Omega(DM) puzzle, Phys. Lett. B 605 (2005) 228 [hep-ph/0410114] [InSPIRE].

[24] C. Arina and N. Fornengo, Sneutrino cold dark matter, a new analysis: Relic abundance and detection rates, JHEP 11 (2007) 029 [arXiv:0709.4477] [INSPIRE].

[25] Z. Thomas, D. Tucker-Smith and N. Weiner, Mixed Sneutrinos, Dark Matter and the CERN LHC, Phys. Rev. D 77 (2008) 115015 [arXiv:0712.4146] [INSPIRE].

[26] G. Bélanger, S. Kraml and A. Lessa, Light Sneutrino Dark Matter at the LHC, JHEP 07 (2011) 083 [arXiv: 1105.4878] [INSPIRE].

[27] C. Arina and M.E. Cabrera, Multi-lepton signatures at LHC from sneutrino dark matter, JHEP 04 (2014) 100 [arXiv: 1311.6549] [INSPIRE].

[28] P.S. Bhupal Dev, S. Mondal, B. Mukhopadhyaya and S. Roy, Phenomenology of Light Sneutrino Dark Matter in cMSSM/mSUGRA with Inverse Seesaw, JHEP 09 (2012) 110 [arXiv: 1207.6542] [INSPIRE].

[29] J. Guo, Z. Kang, J. Li, T. Li and Y. Liu, Simplified Supersymmetry with Sneutrino LSP at 8 TeV LHC, JHEP 10 (2014) 164 [arXiv:1312.2821] [INSPIRE].

[30] L.A. Harland-Lang, C.-H. Kom, K. Sakurai and M. Tonini, Sharpening $m_{T 2}$ cusps: the mass determination of semi-invisibly decaying particles from a resonance, JHEP 06 (2014) 175 [arXiv: 1312.5720] [INSPIRE].

[31] LUX collaboration, D.S. Akerib et al., First results from the LUX dark matter experiment at the Sanford Underground Research Facility, Phys. Rev. Lett. 112 (2014) 091303 [arXiv:1310.8214] [INSPIRE]. 
[32] J. Alwall, P. Schuster and N. Toro, Simplified Models for a First Characterization of New Physics at the LHC, Phys. Rev. D 79 (2009) 075020 [arXiv: 0810.3921] [INSPIRE].

[33] LhC New Physics Working Group collaboration, D. Alves et al., Simplified Models for LHC New Physics Searches, J. Phys. G 39 (2012) 105005 [arXiv:1105.2838] [InSPIRE].

[34] S. Kraml et al., SModelS: a tool for interpreting simplified-model results from the LHC and its application to supersymmetry, Eur. Phys. J. C 74 (2014) 2868 [arXiv:1312.4175] [INSPIRE].

[35] S. Kraml et al., SModelS v1.0: a short user guide, arXiv:1412.1745 [INSPIRE].

[36] http://smodels.hephy.at.

[37] D. Tucker-Smith and N. Weiner, Inelastic dark matter, Phys. Rev. D 64 (2001) 043502 [hep-ph/0101138] [INSPIRE].

[38] Y. Grossman and H.E. Haber, Sneutrino mixing phenomena, Phys. Rev. Lett. 78 (1997) 3438 [hep-ph/9702421] [INSPIRE].

[39] LHCb collaboration, First Evidence for the Decay $B_{s}^{0} \rightarrow \mu^{+} \mu^{-}$, Phys. Rev. Lett. 110 (2013) 021801 [arXiv: 1211.2674] [INSPIRE].

[40] Heavy Flavor Averaging Group collaboration, Y. Amhis et al., Averages of B-Hadron, C-Hadron and tau-lepton properties as of early 2012, arXiv:1207.1158 [INSPIRE].

[41] Particle Data Group collaboration, K. Olive et al., Review of Particle Physics, Chin. Phys. C 38 (2014) 090001.

[42] G. Bélanger, B. Dumont, U. Ellwanger, J.F. Gunion and S. Kraml, Global fit to Higgs signal strengths and couplings and implications for extended Higgs sectors, Phys. Rev. D 88 (2013) 075008 [arXiv: 1306 . 2941] [INSPIRE].

[43] LEP2 SUSY Working Group, ALEPH, DELPHI, L3, OPAL collaborations, Combined LEP Selectron/Smuon/Stau Results, 183-208 GeV, LEPSUSYWG/04-01.1.

[44] D0 collaboration, V.M. Abazov et al., Search for squarks and gluinos in events with jets and missing transverse energy using $2.1 \mathrm{fb}^{-1}$ of pp collision data at $\sqrt{s}=1.96 \mathrm{TeV}$, Phys. Lett. B 660 (2008) 449 [arXiv:0712.3805] [INSPIRE].

[45] M.E. Cabrera, J.A. Casas and R.R. de Austri, The health of SUSY after the Higgs discovery and the XENON100 data, JHEP 07 (2013) 182 [arXiv:1212.4821] [INSPIRE].

[46] B.C. Allanach, A. Djouadi, J.L. Kneur, W. Porod and P. Slavich, Precise determination of the neutral Higgs boson masses in the MSSM, JHEP 09 (2004) 044 [hep-ph/0406166] [INSPIRE].

[47] ALEPH, DELPHI, L3, OPAL, SLD collaborations, LEP Electroweak Working Group, SLD Electroweak Group, SLD Heavy Flavour Group, S. Schael et al., Precision electroweak measurements on the $Z$ resonance, Phys. Rept. 427 (2006) 257 [hep-ex/0509008] [InSPIRE].

[48] F. Boudjema, G. Drieu La Rochelle and S. Kulkarni, One-loop corrections, uncertainties and approximations in neutralino annihilations: Examples, Phys. Rev. D 84 (2011) 116001 [arXiv: 1108.4291] [INSPIRE].

[49] B.C. Allanach, SOFTSUSY: a program for calculating supersymmetric spectra, Comput. Phys. Commun. 143 (2002) 305 [hep-ph/0104145] [INSPIRE].

[50] C. Duhr and B. Fuks, A superspace module for the FeynRules package, Comput. Phys. Commun. 182 (2011) 2404 [arXiv:1102.4191] [INSPIRE]. 
[51] C. Degrande et al., UFO - The Universal FeynRules Output, Comput. Phys. Commun. 183 (2012) 1201 [arXiv: 1108. 2040] [INSPIRE].

[52] G. Bélanger, F. Boudjema, A. Pukhov and A. Semenov, MicrOMEGAs_3: A program for calculating dark matter observables, Comput. Phys. Commun. 185 (2014) 960 [arXiv: 1305.0237] [INSPIRE].

[53] F. Mahmoudi, SuperIso v2.3: A program for calculating flavor physics observables in Supersymmetry, Comput. Phys. Commun. 180 (2009) 1579 [arXiv:0808.3144] [INSPIRE].

[54] F. Feroz and M.P. Hobson, Multimodal nested sampling: an efficient and robust alternative to MCMC methods for astronomical data analysis, Mon. Not. Roy. Astron. Soc. 384 (2008) 449 [arXiv: 0704.3704] [inSPIRE].

[55] F. Feroz, M.P. Hobson and M. Bridges, MultiNest: an efficient and robust Bayesian inference tool for cosmology and particle physics, Mon. Not. Roy. Astron. Soc. 398 (2009) 1601 [arXiv:0809.3437] [INSPIRE].

[56] F. Feroz, M.P. Hobson, E. Cameron and A.N. Pettitt, Importance Nested Sampling and the MultiNest Algorithm, arXiv:1306.2144 [INSPIRE].

[57] T. Sjöstrand, S. Mrenna and P.Z. Skands, PYTHIA 6.4 Physics and Manual, JHEP 05 (2006) 026 [hep-ph/0603175] [INSPIRE].

[58] W. Beenakker, R. Hopker, M. Spira and P.M. Zerwas, Squark and gluino production at hadron colliders, Nucl. Phys. B 492 (1997) 51 [hep-ph/9610490] [INSPIRE].

[59] W. Beenakker, M. Krämer, T. Plehn, M. Spira and P.M. Zerwas, Stop production at hadron colliders, Nucl. Phys. B 515 (1998) 3 [hep-ph/9710451] [INSPIRE].

[60] A. Kulesza and L. Motyka, Threshold resummation for squark-antisquark and gluino-pair production at the LHC, Phys. Rev. Lett. 102 (2009) 111802 [arXiv:0807.2405] [INSPIRE].

[61] A. Kulesza and L. Motyka, Soft gluon resummation for the production of gluino-gluino and squark-antisquark pairs at the LHC, Phys. Rev. D 80 (2009) 095004 [arXiv:0905.4749] [INSPIRE].

[62] W. Beenakker et al., Soft-gluon resummation for squark and gluino hadroproduction, JHEP 12 (2009) 041 [arXiv: 0909.4418] [INSPIRE].

[63] W. Beenakker et al., Supersymmetric top and bottom squark production at hadron colliders, JHEP 08 (2010) 098 [arXiv: 1006.4771] [INSPIRE].

[64] W. Beenakker et al., Squark and Gluino Hadroproduction, Int. J. Mod. Phys. A 26 (2011) 2637 [arXiv: 1105.1110] [inSPIRE].

[65] A. Buckley, PySLHA: a Pythonic interface to SUSY Les Houches Accord data, arXiv: 1305.4194 [INSPIRE].

[66] P.Z. Skands et al., SUSY Les Houches accord: Interfacing SUSY spectrum calculators, decay packages and event generators, JHEP 07 (2004) 036 [hep-ph/0311123] [INSPIRE].

[67] Extending the SLHA: cross section information, http://phystev.in2p3.fr/wiki/2013:groups:tools:slha.

[68] ATLAS collaboration, Search for new phenomena in final states with large jet multiplicities and missing transverse momentum at $\sqrt{s}=8$ TeV proton-proton collisions using the ATLAS experiment, JHEP 10 (2013) 130 [arXiv:1308.1841] [INSPIRE]. 
[69] ATLAS collaboration, Search for squarks and gluinos with the ATLAS detector in final states with jets and missing transverse momentum using $\sqrt{s}=8$ TeV proton-proton collision data, JHEP 09 (2014) 176 [arXiv:1405.7875] [InSPIRE].

[70] ATLAS collaboration, Search for strong production of supersymmetric particles in final states with missing transverse momentum and at least three b-jets using $20.1 \mathrm{fb}^{-} 1 \mathrm{of} p \mathrm{pp}$ collisions at $\sqrt{s}=8 \mathrm{TeV}$ with the ATLAS Detector., ATLAS-CONF-2013-061 (2013).

[71] CMS collaboration, Search for gluino mediated bottom- and top-squark production in multijet final states in pp collisions at 8 TeV, Phys. Lett. B 725 (2013) 243 [arXiv:1305.2390] [INSPIRE].

[72] CMS collaboration, Search for supersymmetry in hadronic final states with missing transverse energy using the variables $\alpha_{T}$ and b-quark multiplicity in pp collisions at $\sqrt{s}=8 \mathrm{TeV}$, Eur. Phys. J. C 73 (2013) 2568 [arXiv:1303.2985] [INSPIRE].

[73] CMS collaboration, Search for supersymmetry in pp collisions at $\sqrt{s}=8 \mathrm{TeV}$ in events with a single lepton, large jet multiplicity and multiple b jets, Phys. Lett. B 733 (2014) 328 [arXiv: 1311.4937] [INSPIRE].

[74] CMS collaboration, Search for new physics in the multijet and missing transverse momentum final state in proton-proton collisions at $\sqrt{s}=8 \mathrm{TeV}$, JHEP 06 (2014) 055 [arXiv: 1402.4770] [INSPIRE].

[75] ATLAS collaboration, Search for direct production of charginos, neutralinos and sleptons in final states with two leptons and missing transverse momentum in pp collisions at $\sqrt{s}=8 \mathrm{TeV}$ with the ATLAS detector, JHEP 05 (2014) 071 [arXiv:1403.5294] [INSPIRE].

[76] CMS collaboration, Searches for electroweak production of charginos, neutralinos and sleptons decaying to leptons and $W, Z$ and Higgs bosons in pp collisions at $8 \mathrm{TeV}$, Eur. Phys. J. C 74 (2014) 3036 [arXiv: 1405.7570] [INSPIRE].

[77] ATLAS collaboration, Search for the direct production of charginos, neutralinos and staus in final states with at least two hadronically decaying taus and missing transverse momentum in pp collisions at $\sqrt{s}=8 \mathrm{TeV}$ with the ATLAS detector, JHEP 1410 (2014) 96 [arXiv: 1407.0350] [INSPIRE].

[78] ATLAS collaboration, Search for new particles in events with one lepton and missing transverse momentum in pp collisions at $\sqrt{s}=8 \mathrm{TeV}$ with the ATLAS detector, JHEP 09 (2014) 037 [arXiv: 1407.7494] [INSPIRE].

[79] CMS collaboration, Search for physics beyond the standard model in final states with a lepton and missing transverse energy in proton-proton collisions at $\sqrt{s}=8 \mathrm{TeV}$, arXiv: 1408.2745 [INSPIRE].

[80] XENON1T collaboration, E. Aprile, The XENON1T Dark Matter Search Experiment, Springer Proc. Phys. C12-02-22 (2013) 93 [arXiv:1206.6288] [INSPIRE].

[81] J. Billard, L. Strigari and E. Figueroa-Feliciano, Implication of neutrino backgrounds on the reach of next generation dark matter direct detection experiments, Phys. Rev. D 89 (2014) 023524 [arXiv: 1307.5458 ] [INSPIRE].

[82] M. Drees, H. Dreiner, D. Schmeier, J. Tattersall and J.S. Kim, CheckMATE: Confronting your Favourite New Physics Model with LHC Data, Comput. Phys. Commun. 187 (2014) 227 [arXiv: 1312.2591] [INSPIRE]. 
[83] B. Dumont et al., Toward a public analysis database for LHC new physics searches using MADANALYSIS 5, Eur. Phys. J. C 75 (2015) 56 [arXiv:1407.3278] [inSPIRE].

[84] S. Kraml et al., Searches for New Physics: Les Houches Recommendations for the Presentation of LHC Results, Eur. Phys. J. C 72 (2012) 1976 [arXiv:1203.2489] [InSPIRE].

[85] B. Dumont, MadAnalysis 5 implementation of ATLAS-SUSY-2013-11: di-leptons plus MET, (2014) [doi:10.7484/INSPIREHEP.DATA.HLMR.T56W.2].

[86] J. Alwall, M. Herquet, F. Maltoni, O. Mattelaer and T. Stelzer, MadGraph 5: Going Beyond, JHEP 06 (2011) 128 [arXiv:1106.0522] [InSPIRE].

[87] J. Alwall et al., The automated computation of tree-level and next-to-leading order differential cross sections and their matching to parton shower simulations, JHEP 07 (2014) 079 [arXiv: 1405.0301] [INSPIRE].

[88] DELPHES 3 collaboration, J. de Favereau et al., DELPHES 3, A modular framework for fast simulation of a generic collider experiment, JHEP 02 (2014) 057 [arXiv:1307.6346] [INSPIRE].

[89] C.G. Lester and D.J. Summers, Measuring masses of semiinvisibly decaying particles pair produced at hadron colliders, Phys. Lett. B 463 (1999) 99 [hep-ph/9906349] [InSPIRE].

[90] H.-C. Cheng and Z. Han, Minimal Kinematic Constraints and m(T2), JHEP 12 (2008) 063 [arXiv: 0810.5178] [INSPIRE].

[91] CMS collaboration, Searches for long-lived charged particles in pp collisions at $\sqrt{s}=7$ and $8 \mathrm{TeV}$, JHEP 07 (2013) 122 [arXiv:1305.0491] [INSPIRE].

[92] ATLAS collaboration, Searches for heavy long-lived charged particles with the ATLAS detector in proton-proton collisions at $\sqrt{s}=8 \mathrm{TeV}$, JHEP 01 (2015) 068 [arXiv:1411.6795] [INSPIRE].

[93] ATLAS collaboration, Search for long-lived stopped R-hadrons decaying out-of-time with pp collisions using the ATLAS detector, Phys. Rev. D 88 (2013) 112003 [arXiv:1310.6584] [INSPIRE].

[94] ATLAS collaboration, Limits on metastable gluinos from ATLAS SUSY searches at 8 TeV, ATLAS-CONF-2014-037 (2014).

[95] A. Arvanitaki, C. Davis, P.W. Graham, A. Pierce and J.G. Wacker, Limits on split supersymmetry from gluino cosmology, Phys. Rev. D 72 (2005) 075011 [hep-ph/0504210] [INSPIRE]. 